\title{
Weak Solutions to a Fluid-Structure Interaction Model of a Viscous Fluid with an Elastic Plate under Coulomb Friction Coupling
}

\author{
Reinhard Farwig * and Andreas Schmidt
}

check for updates

Citation: Farwig, R.; Schmidt, A. Weak Solutions to a Fluid-Structure Interaction Model of a Viscous Fluid with an Elastic Plate under Coulomb Friction Coupling. Mathematics 2021, 9, 1026. https://doi.org/10.3390/ math9091026

Academic Editor: Yoshihiro Shibata

Received: 29 March 2021

Accepted: 28 April 2021

Published: 1 May 2021

Publisher's Note: MDPI stays neutral with regard to jurisdictional claims in published maps and institutional affiliations.

Copyright: (c) 2021 by the authors. Licensee MDPI, Basel, Switzerland. This article is an open access article distributed under the terms and conditions of the Creative Commons Attribution (CC BY) license (https:/ / creativecommons.org/licenses/by/ $4.0 /)$.
Department of Mathematics, University of Technology, 64289 Darmstadt, Germany; aschmidt@mathematik.tu-darmstadt.de

* Correspondence: farwig@mathematik.tu-darmstadt.de

Abstract: We consider the behavior of a viscous fluid within a container that has an elastic upper, free boundary. The movement of the upper boundary is described by a combination of a plate equation and a boundary condition of friction type that quantifies the elasticity of the boundary. We show the local existence of weak solutions to this coupled system in three dimensions, by applying the Galerkin method to a regularized version of the problem and using a fixed-point argument afterwards.

Keywords: Kirchhoff-type plate equation; boundary condition of friction type; coupled system

\section{Introduction}

Let $\omega$ denote a bounded Lipschitz domain in $\mathbb{R}^{2}$. We consider a domain,

$$
\Omega_{h_{0}}=\left\{(x, y, z) \in \mathbb{R}^{3} \mid(x, y) \in \omega, 0<z<1+h_{0}(x, y)\right\},
$$

filled with a viscous fluid, where $h_{0}$ is a given vertical displacement of the elastic, upper part of the boundary from the reference state $\omega \times\{1\}$. We denote the rigid part of the boundary of $\Omega_{h_{0}}$ by $\Gamma_{0}:=(\omega \times\{0\}) \cup(\partial \omega \times[0,1])$, which consists of the lower part of the boundary as well as of the lateral part. Only $\Gamma_{h_{0}}=\partial \Omega_{h_{0}} \backslash \Gamma_{0}$, the upper part of $\partial \Omega_{h_{0}}$, is subject to change in time which is described by a function $h$ that is to be determined. So, while $\Omega_{h_{0}}$ denotes the domain occupied by the fluid at the initial time $t=0$,

$$
\Omega_{h(t)}=\left\{(x, y, z) \in \mathbb{R}^{3} \mid(x, y) \in \omega, 0<z<1+h(t, x, y)\right\}
$$

is the domain occupied by the fluid at time $t$. The equations that describe the interplay between the fluid flow and the elastic boundary, are expressed by $(u, p)$ and $h$, respectively. They are defined on the non-cylindrical open space-time domain

$$
\widehat{\Omega}_{h}:=\bigcup_{t \in(0, T)}\{t\} \times \Omega_{h(t)}
$$

and satisfy the coupled non-linear system

$$
\begin{aligned}
\partial_{t} u-\operatorname{div}(2 \mu D(u)-p I) & =f-(u \cdot \nabla) u & & \text { in } \widehat{\Omega}_{h}, \\
\operatorname{div} u & =0 & & \text { in } \widehat{\Omega}_{h}, \\
u(t, \cdot) & =0 & & \text { on } \Gamma_{0,} \\
u(t, x, y, 1+h(t, x, y)) & =\left(0,0, \partial_{t} h(t, x, y)\right)^{\top} & & \text { on }(0, T) \times \omega, \\
h & =\frac{\partial h}{\partial \bar{n}_{\omega}}=0 & & \text { on } \partial \omega,
\end{aligned}
$$




$$
\begin{aligned}
u_{\mid t=0} & =u_{0} & & \text { in } \Omega_{h_{0}} \\
\left(h, \partial_{t} h\right)_{\mid t=0} & =\left(h_{0}, h_{1}\right) & & \text { in } \omega,
\end{aligned}
$$

with $D u:=\frac{1}{2}\left(\nabla u+\nabla u^{\top}\right)$ and $\bar{n}_{\omega} \in \mathbb{R}^{2}$ denoting the outer unit normal on $\partial \omega$. On $\omega \times(0, T)$ we have for given $\lambda, \kappa>0$ that

$$
\partial_{t} h= \begin{cases}0, & \text { if }\left|\partial_{t}^{2} h+\Delta^{2} h+\lambda \Delta^{2} \partial_{t} h-\left(T_{f \ell}\right)_{3}\right|<\kappa \\ -\alpha\left(\partial_{t}^{2} h+\Delta^{2} h+\lambda \Delta^{2} \partial_{t} h-\left(T_{f \ell}\right)_{3}\right), & \text { if }\left|\partial_{t}^{2} h+\Delta^{2} h+\lambda \Delta^{2} \partial_{t} h-\left(T_{f \ell}\right)_{3}\right|=\kappa\end{cases}
$$

for $\alpha=\alpha(t, x, y) \geq 0$ which is equivalent to

$$
\left(\partial_{t}^{2} h+\Delta^{2} h+\lambda \Delta^{2} \partial_{t} h-\left(T_{f \ell}\right)_{3}\right) \eta \geq \kappa\left|\partial_{t} h\right|-\kappa\left|\partial_{t} h+\eta\right| \forall \eta \in \mathbb{R} .
$$

Here, $\alpha=\frac{\left|\partial_{t} h\right|}{\kappa}$ in (9) 2 and $\alpha=0$ in case (9) $)_{1}$ is an unknown function of the system; furthermore, $\mu>0$ denotes the viscosity of the fluid. Equations (2) and (3) are the incompressible Navier-Stokes equations with a given external force $f$, where (3) and (5) imply that the flow is volume preserving; see Remark 1 (ii). Moreover, (4), describes that the fluid does not move on the rigid part $\Gamma_{0}$. Since the fluid adheres to the plate, we postulate the kinematic boundary condition (5). Moreover, $T_{f \ell}=T_{f \ell}(t, x, y)$ is the surface force exerted by the fluid on the structure given by

$$
\int_{\omega} T_{f \ell}(t, x, y) \cdot v(t, x, y, 1+h(t, x, y)) d x d y=\int_{\Gamma_{h(t)}}\left(-2 \mu D(u) \cdot n_{t}+p n_{t}\right) \cdot v d \sigma
$$

for all $v \in C_{c}^{\infty}\left(\bar{\Omega}_{h(t)}\right)^{3}$, where $n_{t}=\frac{\left(-\nabla^{\prime} h, 1\right)^{\top}}{\sqrt{1+\left|\nabla^{\prime} h\right|^{2}}}$ is the $t$-dependent outer normal at $\Gamma_{h(t)}$. Instead of the boundary condition (9), often an equation of the form

$$
\partial_{t}^{2} h+\Delta^{2} h+\lambda \Delta^{2} \partial_{t} h=\left(T_{f \ell}\right)_{3}+g
$$

is used in the literature. For instance, in Reference [1], Chambolle et al. have constructed weak solutions for the corresponding problem; the authors remark that the damping term $\Delta^{2} \partial_{t} h$ can be replaced by the term $\Delta \partial_{t}^{2} h$. Similar results have been shown by Grandmont [2] in the case of a three-dimensional cavity, where one part of the boundary is assumed to be elastic and the other one rigid. However, the focus in Reference [2] is on the behavior of solutions in the limit $\lambda \rightarrow 0$ and a uniform positive lower bound of the time of existence.

In Reference [3], Denk and Saal exploit the damping term $-\Delta \partial_{t} h$ in a half space like domain with base $\omega=\mathbb{R}^{n-1}$ and maximal regularity to get strong solutions; similar techniques are used by Celik and Kyed [4] in a spatially periodic setting $(n=3)$ to prove the existence of strong $t$-periodic solutions. Beiraõ da Veiga [5] proved the local existence of strong solutions in a one-dimensional periodic base $\omega=(0, L)$. Similar results are obtained by Badra and Takahashi [6] working with semigroups of Gevrey class. Fluid structure interaction problems with a classical non-linear von Kármán shallow shell of finite thickness that allow for both transversal and lateral displacements are considered by Chueshov and Ryzhkova [7]. Lengeler and Ružička [8] discussed the case of a Koiter shell instead of the flat plate and, hence, replaced the operator $\Delta^{2}$ by an operator better suited for non-flat boundaries.

These equations model the movement of the plate via a (damped) plate equation, where the damping term used here is $\lambda \Delta^{2} \partial_{t} h$ and the forces causing the movement are given by $\left(T_{f \ell}\right)_{3}$ and a prescribed external force $g$. Our boundary condition is a combination of this equation with a boundary condition of friction type, namely (for $h=0$ )

$$
u_{n}=\left\{\begin{array}{ll}
0, & \text { if }\left|\left(T_{f \ell}\right)_{n}\right|<\kappa \\
-\alpha\left(T_{f \ell}\right)_{n}, & \text { if }\left|\left(T_{f \ell}\right)_{n}\right|=\kappa \text { with } \alpha \geq 0
\end{array} \text { and } \quad u_{\tau}=0\right.
$$


Here, the subscripts $n$ and $\tau$ denote the normal and tangential component of a vector, respectively.

Condition (12) describes that the fluid adheres to the boundary, but if the normal component of the stress tensor becomes large enough, the fluid may start to "leak" and presses the upper elastic boundary in the opposite direction of $\left(T_{f \ell}\right)_{n}$. This boundary condition allowing for leaking and, in particular, its counterpart on slip where tangential components are controlled as in (12) (Coulomb's boundary or dry friction condition) have been studied extensively. For instance, the existence of strong solutions with respect to time for the Navier-Stokes equations coupled with this boundary condition, i.e., $u \in W_{2}^{1}\left(0, T ; L^{2}(\Omega)\right)$, has been shown by Kashiwabara [9] for finite time intervals. On the other hand, a thorough analysis of the stationary Stokes case in bounded smooth domains of $\mathbb{R}^{d}, d \geq 3$, including $H^{2}$-results and the Stokes resolvent problem, is due to Saito [10]. Besides results on weak solutions, Bălilescu, San Martín, and Takahashi [11] present several numerical examples for the slip Coulomb boundary condition, whereas Jing et al. [12] propose a discontinuous Galerkin method for the non-linear leak boundary condition. In [13], Bălilescu et al. consider the translational and rotational motion of a rigid body immersed into a viscous fluid together with Coulomb's boundary condition. Consiglieri [14] discusses the stationary case of a non-Newtonian fluid with Coulomb's condition, whereas Baranovskii [15] puts the focus on a viscoelastic fluids of Oldroyd-B type.

In combination, the boundary condition (9) describes that, if the force $g:=\partial_{t}^{2} h+$ $\Delta^{2} h+\lambda \Delta^{2} \partial_{t} h-\left(T_{f \ell}\right)_{3}$ is smaller than the threshold $\kappa$, then $h$, and, therefore, the domain does not change in time. However, if the force becomes large enough, the upper boundary may start to change in time, and it does so in the opposite direction of the force. So, $\kappa$ can be viewed as a constant measuring the elasticity of the plate, which is the upper boundary. If the material that the plate consists of is very elastic, $\kappa$ is very small. If $\kappa$ is very large, the plate is harder to move. For simplicity, we assume in the following that $\lambda=1$.

In this work, we show the existence of weak solutions. First, we will formally derive an energy estimate to identify suitable solution spaces in Section 2. In Section 3, we rigorously define the solution spaces and gather results about trace operators associated with the domain $\Omega_{h(t)}$. In Section 4, we define weak solutions before we show their existence in Sections 5 and 6. Indeed, in Section 5, we describe several smoothing arguments and perform the Galerkin approximation method on a given domain $\widetilde{\Omega}_{\delta(t)}$, yielding a weak solution $(u, h)$ which ignores the kinematic boundary condition (5) on the upper part of the boundary. Finally, in Section 6, a fixed point argument yields a solution $u$ satisfying $h=\delta$, i.e., the kinematic boundary condition and also (9).

We want to emphasize that we follow the work of Chambolle, Desjardins, Esteban, and Grandmont; see Reference [1]; however, we have an additional non-linear boundary term. In a first weak formulation, as in (19) or (20) in Section 4, we get a variational inequality in which both the solution and the test function are variables in the non-linear function $|\cdot|$. By a mollification, the modulus is replaced by a smooth function $G_{\varepsilon}$, as in (28) below, which is non-linear in the solution, but linear in the test function. These terms require from time to time significant changes from the mentioned paper. In order to keep this work self-contained and comprehensible, we still give a detailed proof of the existence of weak solution that is in some points even more detailed than that in [1].

\section{Energy Estimate}

Formally multiplying (2) with $u$ and integrating over $\Omega_{h}$ leads to

$$
\begin{aligned}
& \int_{\Omega_{h(t)}} \partial_{t} u \cdot u+\int_{\Omega_{h}(t)}(u \cdot \nabla) u \cdot u+2 \mu \int_{\Omega_{h(t)}}|D(u)|^{2} \\
& +\int_{\Gamma_{h(t)}}\left(-2 \mu D(u) \cdot n_{t}+p n_{t}\right) \cdot u=\int_{\Omega_{h(t)}} f \cdot u .
\end{aligned}
$$


Note that we will omit the infinitesimal increments, such as $d x d y$ in $\mathbb{R}^{2}, d \sigma$ for $2 \mathrm{~d}$-surfaces, and $d \tau$ for time to keep lengthy formulae short. Then, the Reynolds transport theorem, as in [16] (Theorem 3.5), implies that

$$
\frac{d}{d t} \int_{\Omega_{h(t)}} \frac{1}{2}|u|^{2}=\int_{\Omega_{h(t)}} \frac{d}{d t}\left(\frac{1}{2}|u|^{2}\right)+\int_{\partial \Omega_{h(t)} \backslash \Gamma_{0}} \frac{1}{2}|u|^{2} v \cdot n_{t},
$$

with $v$ denoting the velocity of the area element which equals $u$. Hence, using $\operatorname{div} u=0$ and the divergence theorem, we deduce that

$$
\int_{\Omega_{h}(t)}(u \cdot \nabla) u \cdot u=\frac{1}{2} \int_{\partial \Omega_{h(t)}}|u|^{2} u \cdot n_{t}=\frac{d}{d t} \int_{\Omega_{h(t)}} \frac{1}{2}|u|^{2}-\int_{\Omega_{h(t)}} \frac{d}{d t}\left(\frac{1}{2}|u|^{2}\right) .
$$

Plugging this into (13), we get that

$$
\frac{1}{2} \frac{d}{d t} \int_{\Omega_{h(t)}}|u|^{2}+2 \mu \int_{\Omega_{h(t)}}|D(u)|^{2}+\int_{\Gamma_{h(t)}}\left(-2 \mu D(u) \cdot n_{t}+p n_{t}\right) \cdot u=\int_{\Omega_{h(t)}} f \cdot u .
$$

Now, due to the definition of $T_{f \ell}$ given by (11), we calculate for the boundary term

$$
\begin{aligned}
& \int_{\partial \Omega_{h(t)} \Gamma_{0}}\left(-2 \mu D(u) \cdot n_{t}+p n_{t}\right) \cdot u=\int_{\omega} T_{f \ell} \cdot u(t, x, y, 1+h(t, x, y)) \\
& \stackrel{(5)}{=} \int_{\omega}\left(T_{f \ell}\right)_{3} \partial_{t} h=\int_{\omega}-\left(\partial_{t}^{2} h+\Delta^{2} h+\mu \Delta^{2} \partial_{t} h-\left(T_{f \ell}\right)_{3}\right) \partial_{t} h \\
& \quad+\int_{\omega}\left(\partial_{t}^{2} h+\Delta^{2} h+\mu \Delta^{2} \partial_{t} h\right) \partial_{t} h .
\end{aligned}
$$

We rewrite the last integral as

$$
\int_{\omega}\left(\frac{1}{2} \frac{d}{d t}\left|\partial_{t} h\right|^{2}+\frac{1}{2} \frac{d}{d t}|\Delta h|^{2}+\mu\left|\Delta \partial_{t} h\right|^{2}\right) .
$$

Applying (10) to the second last integral, we get

$$
\int_{\omega}-\left(\partial_{t}^{2} h+\Delta^{2} h+\mu \Delta^{2} \partial_{t} h-\left(T_{f \ell}\right)_{3}\right) \partial_{t} h \leq \int_{\omega}\left(\kappa\left|2 \partial_{t} h\right|-\kappa\left|\partial_{t} h\right|\right)=\int_{\omega} \kappa\left|\partial_{t} h\right| .
$$

We also obtain the reverse inequality if we consider $y=-\partial_{t} h$ in (10). Hence, we conclude

$$
\int_{\omega}-\left(\partial_{t}^{2} h+\Delta^{2} h+\mu \Delta^{2} \partial_{t} h-()_{3}\right) \partial_{t} h=\int_{\omega} \kappa\left|\partial_{t} h\right| .
$$

Plugging these identities into (14), we get the equality

$$
\begin{aligned}
& \frac{1}{2} \frac{d}{d t} \int_{\Omega_{h(t)}}|u|^{2}+2 \mu \int_{\Omega_{h(t)}}|D(u)|^{2}+\int_{\omega}\left(\frac{1}{2} \frac{d}{d t}\left|\partial_{t} h\right|^{2}+\frac{1}{2} \frac{d}{d t}|\Delta h|^{2}+\mu\left|\Delta \partial_{t} h\right|^{2}+\kappa\left|\partial_{t} h\right|\right) \\
& \quad=\int_{\Omega_{h(t)}} f \cdot u .
\end{aligned}
$$

Integrating over time yields for all $t>0$

$$
\begin{aligned}
& \frac{1}{2} \int_{\Omega_{h(t)}}|u(t)|^{2}+2 \mu \int_{0}^{t} \int_{\Omega_{h(s)}}|D(u)|^{2} d s+\frac{1}{2} \int_{\omega}\left(\left|\partial_{t} h(t)\right|^{2}+|\Delta h(t)|^{2}\right) \\
& \quad+\int_{0}^{t} \int_{\omega}\left(\mu\left|\Delta \partial_{t} h\right|^{2}+\kappa\left|\partial_{t} h\right|\right) \\
& \quad=\frac{1}{2} \int_{\Omega_{h_{0}}}\left|u_{0}\right|^{2}+\frac{1}{2} \int_{\omega}\left(\left|h_{1}\right|^{2}+\left|\Delta h_{0}\right|^{2}\right)+\int_{0}^{t} \int_{\Omega_{h(s)}} f \cdot u .
\end{aligned}
$$


Using Young's and Hölder's inequality, we deduce for all $t>0$

$$
\begin{aligned}
& \frac{1}{2}\left(\|u(t)\|_{L^{2}\left(\Omega_{h(t)}\right)}^{2}+\left\|\partial_{t} h(t)\right\|_{L^{2}(\omega)}^{2}+\frac{1}{2}\|\Delta h(t)\|_{L^{2}(\omega)}^{2}\right) \\
& \quad+2 \mu \int_{0}^{t}\|D(u)\|_{L^{2}\left(\Omega_{h(s)}\right)}^{2} d s+\mu\left\|\Delta \partial_{t} h\right\|_{L^{2}((0, t) \times \omega)}^{2}+\kappa\left\|\partial_{t} h\right\|_{L^{1}((0, t) \times \omega)} \\
& \quad \leq \frac{1}{2} \int_{0}^{t}\|u(s)\|_{L^{2}\left(\Omega_{h(s)}\right)}^{2}+\frac{1}{2}\left(\left\|u_{0}\right\|_{L^{2}\left(\Omega_{h_{0}}\right)}^{2}+\left\|h_{1}\right\|_{L^{2}(\omega)}^{2}+\left\|\Delta h_{0}\right\|_{L^{2}(\omega)}^{2}\right) \\
& \quad+\frac{1}{2} \int_{0}^{t}\|f(s)\|_{L^{2}\left(\Omega_{h(s)}\right)}^{2} d s .
\end{aligned}
$$

Then, Grönwall's lemma yields

$$
\begin{aligned}
& \frac{1}{2}\left(\|u(t)\|_{L^{2}\left(\Omega_{h(t)}\right)}^{2}+\left\|\partial_{t} h(t)\right\|_{L^{2}(\omega)}^{2}+\|\Delta h(t)\|_{L^{2}(\omega)}^{2}\right) \\
& \quad+2 \mu \int_{0}^{t}\|D(u)\|_{L^{2}\left(\Omega_{h(s)}\right)}^{2} d s+\mu\left\|\Delta \partial_{t} h\right\|_{L^{2}((0, t) \times \omega)}^{2}+\kappa\left\|\partial_{t} h\right\|_{L^{1}((0, t) \times \omega)} \\
& \quad \leq \frac{e^{t}}{2}\left(\left\|u_{0}\right\|_{L^{2}\left(\Omega_{h_{0}}\right)}^{2}+\left\|h_{1}\right\|_{L^{2}(\omega)}^{2}+\left\|\Delta h_{0}\right\|_{L^{2}(\omega)}^{2}\right)+\frac{1}{2} \int_{0}^{t}\|f(s)\|_{L^{2}\left(\Omega_{h(s)}\right)}^{2} e^{t-s},
\end{aligned}
$$

for all $t>0$. Hence, it is natural to assume for the given data that $f \in L^{2}\left(0, T ; L^{2}\left(\mathbb{R}^{3}\right)\right)$, $u_{0} \in L^{2}\left(\Omega_{h_{0}}\right), h_{0} \in H_{0}^{2}(\omega)$, and $h_{1} \in L^{2}(\omega)$. Due to the fact that we do not know how large $\Omega_{h(t)}$ is, we defined $f$ on $\mathbb{R}^{3}$, which is not restrictive given that the extension by zero preserves $L^{2}$-integrability. Via (17) these properties, in turn, imply that

$$
\begin{aligned}
& u \in L^{\infty}\left(0, T ; L^{2}\left(\Omega_{h(t)}\right)\right), D(u) \in L^{2}\left(0, T ; L^{2}\left(\Omega_{h(t)}\right)\right), \\
& h \in W^{1, \infty}\left(0, T ; L^{2}(\omega)\right) \cap H^{1}\left(0, T ; H_{0}^{2}(\omega)\right) .
\end{aligned}
$$

For a rigorous definition of these spaces, see the next section. Due to $u(t, \cdot)=0$ on $\Gamma_{0}$, it is tempting to use Korn's inequality in order to get $u \in L^{2}\left(0, T ; H^{1}\left(\Omega_{h(t)}\right)\right)$. However, here, we encounter the main problem in solving Equations (2)-(9) in a weak sense. While $h \in$ $H^{1}\left(0, T ; H_{0}^{2}(\omega)\right) \subset C([0, T] \times \bar{\omega})$ is continuous, $h$ does not need to be Lipschitz continuous. Hence, $\Omega_{h(t)}$ is not necessarily a domain with Lipschitz boundary. Nevertheless, due to the continuity of $h, \Omega_{h(t)}$ is open and uniformly bounded. Therefore, we can find an $M \in \mathbb{N}$ such that $B_{M}:=\omega \times(0, M) \supset \Omega_{h(t)}$ for all $t \in[0, T]$. Now, we define

$$
\bar{u}= \begin{cases}u & \text { in } \Omega_{h(t)}, \\ \left(0,0, \partial_{t} h\right)^{\top} & \text { in } B_{M} \backslash \Omega_{h(t)} .\end{cases}
$$

Due to the interface condition (5) and the previous properties, we see that $D(\bar{u}) \in$ $L^{2}\left((0, T) \times B_{M}\right)$. Ergo, we can apply Korn's inequality in $B_{M}$ to get $\bar{u} \in L^{2}\left(0, T ; H^{1}\left(B_{M}\right)\right)$, and, by restriction, we still conclude that $u \in L^{2}\left(0, T ; H^{1}\left(\Omega_{h(t)}\right)\right)$.

\section{Solution Spaces and Trace Operators}

Let $T>0$ and $0<m<M$. Moreover, let $\delta \in C([0, T] \times \bar{\omega})$ be a function such that

$$
1+\delta(t, x, y) \in[m, M] \text { for all }(t, x, y) \in[0, T] \times \bar{\omega},\left.\delta\right|_{\partial \omega}=0
$$

Then, the set

$$
\Omega_{\delta(t)}:=\left\{(x, y, z) \in \mathbb{R}^{3} \mid(x, y) \in \omega, 0<z<1+\delta(t, x, y) \forall(x, y) \in \omega, z \in \mathbb{R}\right\}
$$


is open and contained in $B_{M}=\omega \times(0, M)$ but does not necessarily have a Lipschitz boundary. Furthermore, we need the open space-time domain $\widehat{\Omega}_{\delta}:=\bigcup_{t \in(0, T)}\{t\} \times \Omega_{\delta(t)}$, cf. (1). Recalling $\Gamma_{0}=(\omega \times\{0\}) \cup(\partial \omega \times[0,1])$, we define the following spaces

$$
\begin{aligned}
& H_{0, \Gamma_{0}}^{1}\left(\Omega_{\delta(t)}\right):=\left\{v \in H^{1}\left(\Omega_{\delta(t)}\right) \mid v_{\mid \Gamma_{0}}=0\right\}, \\
& L^{2}\left(0, T ; H^{1}\left(\Omega_{\delta(t)}\right)\right):=\left\{v \in L^{2}\left(\widehat{\Omega}_{\delta}\right) \mid \nabla v \in L^{2}\left(\hat{\Omega}_{\delta}\right)\right\}, \\
& L^{2}\left(0, T ; H_{0}^{1}\left(\Omega_{\delta(t)}\right)\right):=\overline{C_{c}^{\infty}\left(\widehat{\Omega}_{\delta}\right)} L^{2}\left(0, T ; H^{1}\left(\Omega_{\delta(t)}\right)\right), \\
& \mathcal{V}_{\delta}:=\left\{v \in C^{1}\left(\widehat{\Omega}_{\delta}\right) \mid \operatorname{div} v=0, v=0 \text { on }(0, T) \times \Gamma_{0}\right\}, \\
& V_{\delta}:=\overline{\mathcal{V}}_{\delta}^{L^{2}\left(0, T ; H^{1}\left(\Omega_{\delta(t)}\right)\right),} \\
& L^{\infty}\left(0, T ; L^{2}\left(\Omega_{\delta(t)}\right)\right):=\left\{v \in L^{2}\left(\widehat{\Omega}_{\delta}\right) \mid \operatorname{ess} \sup _{t \in(0, T)}\|v\|_{L^{2}\left(\Omega_{\delta(t)}\right)}<\infty\right\}, \\
& V:=\left\{v \in L^{2}\left(0, T ; H^{1}\left(B_{M}\right)\right) \mid \operatorname{div} v=0,\right. \\
&\left.\quad v=0 \text { on }(0, T) \times\left(\Gamma_{0} \cup(\partial \omega \times(1, M))\right)\right\} .
\end{aligned}
$$

Even though $\Omega_{\delta(t)}$ might not necessarily be Lipschitz, one can still show that

$$
V_{\delta}=\left\{v \in L^{2}\left(0, T ; H^{1}\left(\Omega_{\delta(t)}\right)\right) \mid \operatorname{div} v=0, v=0 \text { on }(0, T) \times \Gamma_{0}\right\},
$$

as in [17] (Theorem 3.22, p. 68), by using that $\Omega_{\delta(t)}$ is locally the graph of a function. The following lemmata treat the definition and properties of the trace on $\Gamma_{\delta(t)}$. For these lemmata, always assume that $\delta$ satisfies (18).

Lemma 1. For fixed $t \in[0, T]$, the linear mapping

$$
\gamma_{\delta(t)}: C^{1}\left(\overline{B_{M}}\right) \rightarrow C^{0}(\bar{\omega}), v \mapsto v(x, y, 1+\delta(t, x, y))
$$

can be extended to a linear continuous mapping from $H^{1}\left(B_{M}\right)$ to $L^{2}(\omega)$. Similarly, the same mapping but defined on $C^{1}\left(\bar{\Omega}_{\delta(t)}\right)$ can be extended to $H^{1}\left(\Omega_{\delta(t)}\right)$.

Additionally, we have $\gamma_{\delta(t)}(v) \in L^{2}\left(0, T ; L^{2}(\omega)\right)$ for all $v \in L^{2}\left(0, T ; H^{1}\left(\Omega_{\delta(t)}\right)\right)$.

The proof of this Lemma, as well as the proofs of the following Lemmas 2-6, can be found in Reference [1]. Using the trace operator $\gamma_{\delta(t)}$, we can now rewrite $H_{0}^{1}\left(\Omega_{\delta(t)}\right)$ if we additionally assume that $\delta \in C^{0}\left([0, T] ; H^{1}(\omega)\right)$.

Lemma 2. Additionally, let $\delta \in C^{0}\left([0, T] ; C^{0}(\bar{\omega}) \cap H^{1}(\omega)\right)$, then

$$
H_{0}^{1}\left(\Omega_{\delta(t)}\right)=\left\{v \in H_{0, \Gamma_{0}}^{1}\left(\Omega_{\delta(t)}\right) \mid \gamma_{\delta(t)}(v)=0\right\} .
$$

In particular, $v \in L^{2}\left(0, T ; H_{0}^{1}\left(\Omega_{\delta(t)}\right)\right)$ if and only if $v \in L^{2}\left(0, T ; H_{0, \Gamma_{0}}^{1}\left(\Omega_{\delta(t)}\right)\right)$ and $\gamma_{\delta(t)}(v(t))$ $=0$ for almost all $t \in(0, T)$.

In the next lemma, we consider an extension operator we have already used at the end of the previous section.

Lemma 3. Additionally, let $\delta \in C^{0}\left([0, T] ; C^{0}(\bar{\omega}) \cap H^{1}(\omega)\right)$ and let $b \in L^{2}\left(0, T ; H_{0}^{1}(\omega)\right)$ and $v \in V_{\delta}$ such that $\gamma_{\delta(t)}(v(t))=(0,0, b)^{\top}$ for a.e. $t \in(0, T)$. The function defined by

$$
\bar{v}= \begin{cases}v & \text { in } \widehat{\Omega}_{\delta} \\ (0,0, b)^{\top} & \text { in }\left((0, T) \times B_{M}\right) \backslash \widehat{\Omega}_{\delta}\end{cases}
$$

belongs to $V$ and

$$
\|\bar{v}\|_{V} \leq C\left(\|v\|_{V_{\delta}}+\|b\|_{L^{2}\left(0, T ; H^{1}(\omega)\right)}\right)
$$


where $C>0$ depends only on $M$.

Moreover, we have the existence of a lifting operator:

Lemma 4. For every $\phi \in H_{0}^{1}(\omega)$, there exists a $w \in H_{0, \Gamma_{0}}^{1}\left(\Omega_{\delta(t)}\right)$ such that

$$
\gamma_{\delta(t)}(w)=\phi \quad \text { and } \quad\|w\|_{H^{1}\left(\Omega_{\delta(t)}\right)} \leq C_{m}\|\phi\|_{H^{1}(\omega)} .
$$

The next lemma gives a meaning to the normal trace on the boundary:

Lemma 5. For every $t \in(0, T)$, there exists a linear continuous operator

$$
\gamma_{\delta(t)}^{n}:\left\{v \in L^{2}\left(\Omega_{\delta(t)}\right) \mid \operatorname{div} v \in L^{2}\left(\Omega_{\delta(t)}\right)\right\} \rightarrow\left(H_{0}^{1}(\omega)\right)^{\prime}
$$

with

$$
\gamma_{\delta(t)}^{n}(v)=v(t, x, y, 1+\delta(t, x, y)) \cdot n_{t} \quad \forall(x, y) \in \omega, \forall v \in C^{\infty}\left(\bar{\Omega}_{\delta(t)}\right) .
$$

The following lemma justifies Korn's inequality.

Lemma 6. For all

$$
v, u \in\left\{w \in V_{\delta} \mid \exists b \in L^{2}\left(0, T ; H_{0}^{1}(\omega)\right) \text { s.t. } \gamma_{\delta(t)}(w(t))=(0,0, b)^{\top} \text { for a.e. } t \in(0, T)\right\},
$$

we have

$$
2 \int_{\Omega_{\delta(t)}} D(u): D(v)=\int_{\Omega_{\delta(t)}} \nabla u: \nabla v \text {, for a.e. } t \in(0, T)
$$

thus,

$$
\sqrt{2}\|D(u)\|_{L^{2}\left(\Omega_{\delta(t)}\right)}=\|\nabla u\|_{L^{2}\left(\Omega_{\delta(t)}\right)} \text { for a.e. } t \in(0, T) .
$$

We also have Poincaré's inequality due to the boundedness of $B_{M}$.

Lemma 7. Let $v \in H_{0, \Gamma_{0}}^{1}\left(\Omega_{\delta(t)}\right)$. Then,

$$
\|v\|_{L^{2}\left(\Omega_{\delta(t)}\right)} \leq M\|\nabla v\|_{L^{2}\left(\Omega_{\delta(t)}\right)} .
$$

\section{Weak Formulation}

After the definition of solution and trace spaces and their basic properties, we are now in the position to define weak solutions. Let $u_{0} \in L^{2}\left(\Omega_{h_{0}}\right), h_{1} \in L^{2}(\omega)$ and $h_{0} \in H_{0}^{2}(\omega) \subset$ $C^{0}(\bar{\omega})$.

Definition 1. We call $(u, h)$ a weak solution of (2)-(9) if

(i) $u \in V_{h} \cap L^{\infty}\left(0, T ; L^{2}\left(\Omega_{h(t)}\right)\right)$,

(ii) $h \in W^{1, \infty}\left(0, T ; L^{2}(\omega)\right) \cap H^{1}\left(0, T ; H_{0}^{2}(\omega)\right)$,

(iii) $\gamma_{h(t)}(u(t))=\left(0,0, \partial_{t} h(t)\right)^{\top}$ on $\omega$, for a.e. $t \in(0, T)$, 
(iv) for all test functions $(\phi, b) \in \mathcal{V}_{h} \times C^{1}\left([0, T] ; H_{0}^{2}(\omega)\right)$ satisfying the compatibility condition $\phi(t, x, y, 1+h(t, x, y))=(0,0, b(t, x, y))^{\top}$ for all $(t, x, y) \in[0, T] \times \omega$, there holds the variational inequality

$$
\begin{aligned}
& \int_{\Omega_{h(t)}} u(t) \cdot \phi(t)-\int_{0}^{t} \int_{\Omega_{h(s)}} u \cdot \partial_{t} \phi+2 \mu \int_{0}^{t} \int_{\Omega_{h(s)}} D(u): D(\phi) \\
& +\int_{0}^{t} \int_{\Omega_{h(s)}}(u \cdot \nabla) u \cdot \phi-\int_{0}^{t} \int_{\omega}\left(\partial_{t} h\right)^{2} b+\int_{0}^{t} \int_{\omega} \Delta h \Delta b+\int_{\omega} \partial_{t} h(t) b(t) \\
& -\int_{0}^{t} \int_{\omega} \partial_{t} h \partial_{t} b+\int_{0}^{t} \int_{\omega} \Delta \partial_{t} h \Delta b+\int_{0}^{t} \int_{\omega} \kappa\left(\left|\partial_{t} h\right|-\left|\partial_{t} h-b\right|\right) \\
& \leq \int_{0}^{t} \int_{\Omega_{h(t)}} f \cdot \phi+\int_{\Omega_{h_{0}}} u_{0} \cdot \phi(0)+\int_{\omega} h_{1} b(0)
\end{aligned}
$$

holds for almost all $t \in(0, T)$.

The integral $\int_{0}^{t} \int_{\omega}\left(\partial_{t} h\right)^{2} b$ in (19) is explained by a formal application of Reynolds transport theorem:

$$
\int_{\Omega_{h(t)}} u(t) \phi(t)-\int_{0}^{t} \int_{\Omega_{h(s)}} u \partial_{t} \phi-\int_{\Omega_{h_{0}}} u_{0} \phi(0)=\int_{0}^{t} \int_{\Omega_{h(s)}} \partial_{t} u \phi+\int_{0}^{t} \int_{\omega}\left(\partial_{t} h\right)^{2} b .
$$

To explain the other terms, we formally calculate

$$
\begin{aligned}
& \int_{0}^{t} \int_{\Omega_{h(t)}}(-\operatorname{div}(2 \mu D(u)-p I)) \phi \\
& \quad=2 \mu \int_{0}^{t} \int_{\omega} D(u): D(\phi)+\int_{0}^{t} \int_{\Gamma_{h(t)}}\left(-2 \mu D(u) n_{t}+p n_{t}\right) \phi .
\end{aligned}
$$

Applying (11) to the last term, we estimate

$$
\begin{gathered}
\int_{0}^{t} \int_{\omega}\left(T_{f l}\right)_{3} b=\int_{0}^{t} \int_{\omega}\left(\left(T_{f l}\right)_{3}-\left(\partial_{t}^{2} h+\Delta^{2} h+\Delta^{2} \partial_{t} h\right)\right) b+\int_{0}^{t} \int_{\omega}\left(\partial_{t}^{2} h+\Delta^{2} h+\Delta^{2} \partial_{t} h\right) b \\
\stackrel{(10)}{\geq} \int_{0}^{t} \int_{\omega} \kappa\left(\left|\partial_{t} h\right|-\left|\partial_{t} h-b\right|\right)-\int_{0}^{t} \int_{\omega} \partial_{t} h \partial_{t} b+\int_{\omega} \partial_{t} h(t) b(t) \\
\quad-\int_{\omega} h_{1} b(0)+\int_{0}^{t} \int_{\omega} \Delta h \Delta b+\int_{0}^{t} \int_{\omega} \Delta \partial_{t} h \Delta b .
\end{gathered}
$$

Using the identity

$$
\frac{1}{2} \int_{\Omega_{h(t)}}(u \cdot \nabla) u \cdot \phi=-\frac{1}{2} \int_{\Omega_{h(t)}}(u \cdot \nabla) \phi \cdot u+\frac{1}{2} \int_{\omega}\left(\partial_{t} h\right)^{2} b,
$$

we can also write

$$
\begin{aligned}
\int_{\Omega_{h(t)}} u(t) \cdot \phi(t)-\int_{0}^{t} \int_{\Omega_{h(s)}} u \cdot \partial_{t} \phi+2 \mu \int_{0}^{t} \int_{\Omega_{h(s)}} D(u): D(\phi) \\
\quad+\frac{1}{2} \int_{0}^{t} \int_{\Omega_{h(s)}}(u \cdot \nabla) u \cdot \phi-\frac{1}{2} \int_{0}^{t} \int_{\Omega_{h(s)}}(u \cdot \nabla) \phi \cdot u-\frac{1}{2} \int_{0}^{t} \int_{\omega}\left(\partial_{t} h\right)^{2} b+\int_{0}^{t} \int_{\omega} \Delta h \Delta b \\
\quad+\int_{\omega} \partial_{t} h(t) b(t)-\int_{0}^{t} \int_{\omega} \partial_{t} h \partial_{t} b+\int_{\omega} \int_{0}^{t} \Delta \partial_{t} h \Delta b+\int_{0}^{t} \int_{\omega} \kappa\left(\left|\partial_{t} h\right|-\left|\partial_{t} h-b\right|\right) \\
\leq \int_{0}^{t} \int_{\Omega_{h(t)}} f \cdot \phi+\int_{\Omega_{h_{0}}} u_{0} \cdot \phi(0)+\int_{\omega} h_{1} b(0) .
\end{aligned}
$$

Later, it will become clear why we use (20) rather than (19). 


\section{Existence of Weak Solutions: The Galerkin Approximation}

This section is dedicated to the proof of the main theorem.

Theorem 1. Let $f \in L_{l o c}^{2}\left((0, \infty) ; L^{2}\left(\mathbb{R}^{3}\right)\right), u_{0} \in L^{2}\left(\Omega_{h_{0}}\right), h_{1} \in L^{2}(\omega)$, and $h_{0} \in H_{0}^{2}(\omega)$ with

$$
\begin{aligned}
\min _{\bar{\omega}}\left(1+h_{0}\right) & >0, \\
\operatorname{div} u_{0} & =0 \text { in } \Omega_{h_{0}}, \\
u_{0} \cdot n & =0 \text { on } \Gamma_{0}, \\
\gamma_{h_{0}}^{n}\left(u_{0}\right) & =\left(0,0, h_{1}\right)^{\top} \cdot n_{0} \text { on } \omega, \\
\int_{\omega} h_{1}(x, y) & =0 .
\end{aligned}
$$

Then, there exists a $T^{*} \in(0, \infty]$ and a weak solution $(u, h)$ of $(2)-(9)$ on $[0, T]$ for all $T<T^{*}$. This solution satisfies the estimate

$$
\begin{aligned}
& \|u\|_{L^{\infty}\left(0, T ; L^{2}\left(\Omega_{h(t)}\right)\right)}+\|\nabla u\|_{L^{2}\left(0, T ; L^{2}\left(\Omega_{h(t)}\right)\right)}+\left\|\partial_{t} h\right\|_{L^{\infty}\left(0, T ; L^{2}(\omega)\right)}+\|\Delta h\|_{H^{1}\left(0, T ; L^{2}(\omega)\right)} \\
& \quad \leq C\left(T,\left\|u_{0}\right\|_{L^{2}\left(\Omega_{h_{0}}\right)},\|f\|_{L^{2}\left((0, T) \times \mathbb{R}^{3}\right)},\left\|h_{0}\right\|_{H_{0}^{2}(\omega)},\left\|h_{1}\right\|_{L^{2}(\omega)}\right)
\end{aligned}
$$

with a constant $C>0$.

Remark 1. (i) The first assumption on $h_{0}$, i.e., $(21)_{1}$, is natural since we assume that there is no intersection of the free boundary with the rigid boundary at the start. In fact, the reason why the existence of a global solution for all times will not be shown is due to such a possible self-intersection of the boundary. The other conditions are compatibility conditions. The last one is due to $(21)_{2}$ and $(21)_{4}$.

(ii) The conditions $(21)_{4,5}$ and the solenoidality of $u$ formally imply that the flow is volume preserving. Actually,

$$
\partial_{t} \int_{\omega} h=\int_{\omega} \partial_{t} h=\int_{\Gamma_{h(t)}} u \cdot n_{t}=\int_{\Omega_{h(t)}} \operatorname{div} u=0,
$$

so that $\int_{\omega} h(t)$, the volume of $\Omega_{h(t)}$, equals $\int_{\omega} h_{0}$ for all suitable $t>0$.

Proof. Step 1: The regularization procedure

First, we regularize the problem starting with the initial data. We find a sequence $\left(h_{0}^{\varepsilon}\right)_{\varepsilon>0} \subset C_{c}^{\infty}(\omega)$ such that $\int_{\omega} h_{0}^{\varepsilon}=\int_{\omega} h_{0}$ for all $\varepsilon>0$ and $h_{0}^{\varepsilon} \rightarrow h_{0}$ in $H_{0}^{2}(\omega)$ for $\varepsilon \rightarrow 0$. Since $\min _{\bar{\omega}}\left(1+h_{0}\right)>0$, there is an $m>0$ such that $\min _{\bar{w}}\left(1+h_{0}\right) \geq 2 m>0$. Due to the continuous embedding $H_{0}^{2}(\omega) \hookrightarrow C(\bar{\omega})$, we also have that

$$
\min _{\bar{\omega}}\left(1+h_{0}^{\varepsilon}\right) \geq \frac{3 m}{2}
$$

given that $\varepsilon>0$ is small enough.

Now, we define $\bar{u}_{0}$ by

$$
\bar{u}_{0}= \begin{cases}u_{0} & \text { in } \Omega_{h_{0}} \\ \left(0,0, h_{1}\right)^{\top} & \text { in } B_{M+1} \backslash \Omega_{h_{0}} .\end{cases}
$$

Since $\operatorname{div}\left(u_{0}\right)=0$ in $\Omega_{h_{0}}, \operatorname{div}\left(0,0, h_{1}\right)=0$ in $B_{M+1} \backslash \Omega_{h_{0}}$ and the normal trace $\gamma_{h_{0}}^{n}\left(u_{0}\right)=$ $\left(0,0, h_{1}\right)^{\top} \cdot n_{0}$, we also have that $\operatorname{div}\left(\bar{u}_{0}\right)=0$ in $B_{M+1}$.

For $0<\delta<1$, we define the scaling

$$
\left.u_{0}^{[1+\delta]}(x, y, z)=\left((1+\delta)\left(\bar{u}_{0}\right)_{1},(1+\delta)\left(\bar{u}_{0}\right)_{2},\left(\bar{u}_{0}\right)_{3}\right)(x, y,(1+\delta) z)\right)^{\top}
$$


It is easy to check that $u_{0}^{[1+\delta]}$ is also divergence free. Due to $h_{0} \in C(\bar{\omega})$, the set

$$
\widetilde{Z}:=\left\{(x, y, z) \subset \mathbb{R}^{3} \mid(x, y) \in \omega,(1-\delta / 2)\left(1+h_{0}(x, y)\right)<z<(1+\delta)\left(1+h_{0}(x, y)\right)\right\}
$$

is open and a neighborhood of $\Gamma_{h_{0}}$. In particular, we have that $u_{0}^{[1+\delta]}=\left(0,0, h_{1}\right)^{\top}$ on $\widetilde{Z}$. Due to $h_{0}^{\varepsilon} \rightarrow h_{0}$ in $C(\bar{\omega})$, we get for $\varepsilon>0$ small enough the inclusion $\widetilde{Z}_{\varepsilon} \subset \widetilde{Z}$, where

$$
\widetilde{Z}_{\varepsilon}:=\left\{(x, y, z) \subset \mathbb{R}^{3} \mid(x, y) \in \omega,\left(1-\frac{\delta}{4}\right)\left(1+h_{0}^{\varepsilon}(x, y)\right)<z<\left(1+\frac{\delta}{2}\right)\left(1+h_{0}^{\varepsilon}(x, y)\right)\right\},
$$

a neighborhood $\Gamma_{h_{0}^{\epsilon}}$. Hence, provided that $\varepsilon>0$ is small enough, we also have $u_{0}^{[1+\delta]}=$ $\left(0,0, h_{1}\right)^{\top}$ on $\widetilde{Z}_{\varepsilon}$. Finally, we note the convergence

$$
\chi_{\Omega_{h_{0}^{\delta}}} u_{0}^{[1+\delta]} \rightarrow \chi_{\Omega_{h_{0}}} u_{0} \text { in } L^{2}\left(B_{M}\right) \text { as } \delta \rightarrow 0 .
$$

Now, let $\left(h_{1}^{\varepsilon}\right)_{\varepsilon>0} \subset C^{\infty}(\omega)$ be such that $h_{1}^{\varepsilon} \rightarrow h_{1}$ in $L^{2}(\omega)$ and $\int_{\omega} h_{1}^{\varepsilon}=0$ for all $\varepsilon>0$. Moreover, we can regularize $u_{0}^{[1+\delta]}$ in $L^{2}\left(B_{M+1}\right)$ by functions $\left(u_{0}^{\varepsilon}\right)_{\varepsilon>0} \subset C^{\infty}\left(B_{M+1}\right)$ with $\operatorname{div} u_{0}^{\varepsilon}=0, u_{0}^{\varepsilon}=0$ on $\Gamma_{0}$, and $u_{0}^{\varepsilon}\left(x, y, 1+h_{0}^{\varepsilon}(x, y)\right)=\left(0,0, h_{1}^{\varepsilon}\right)^{\top}$ on $\omega$. The latter can be assumed due to $u_{0}^{[1+\delta]}=\left(0,0, h_{1}\right)^{\top}$ on $\widetilde{Z}_{\mathcal{\varepsilon}}$. In summary, we get that

$$
\begin{aligned}
\operatorname{div} u_{0}^{\varepsilon} & =0, u_{0}^{\varepsilon}\left(x, y, 1+h_{0}^{\varepsilon}(x, y)\right)=\left(0,0, h_{1}^{\varepsilon}\right)^{\top} \text { for all }(x, y) \in \omega, \\
\int_{\omega} h_{1}^{\varepsilon} & =0, u_{0}^{\varepsilon}=0 \text { on } \Gamma_{0}, \quad \int_{\omega} h_{0}^{\varepsilon}=\int_{\omega} h_{0}
\end{aligned}
$$

and the convergences

$$
\begin{array}{rlrl}
\chi_{\Omega_{h_{0}^{\varepsilon}}} u_{0}^{\varepsilon} & \rightarrow \chi_{\Omega_{h_{0}}} u_{0} & & \text { in } L^{2}\left(B_{M}\right), \\
h_{1}^{\varepsilon} & \rightarrow h_{1} & \text { in } L^{2}(\omega), \\
h_{0}^{\varepsilon} & \rightarrow h_{0} & \text { in } H_{0}^{2}(\omega) \text { as } \varepsilon \searrow 0 .
\end{array}
$$

Since the unknown domain $\Omega_{h(t)}$ depends on the solution $h$, we first replace it with a regularised domain $\Omega_{\delta_{\varepsilon}^{\star}(t)}$ and use a fixed point argument in Step 6 below to find $\delta_{\varepsilon}^{\star}=h_{\mathcal{\varepsilon}}$. To be more precise, let $\delta \in H^{1}\left(0, T ; C^{0}(\bar{\omega}) \cap H_{0}^{1}(\omega)\right)$ such that $\delta_{\mid t=0}=h_{0}^{\varepsilon}$ and $M \geq 1+$ $\delta(t, x, y) \geq m>0$ for all $(t, x, y) \in[0, T] \times \bar{\omega}$ with $m$ as before, i.e., $\min _{\bar{\omega}}\left(1+h_{0}\right) \geq 2 m>0$, and $M \in \mathbb{N}$ will be chosen later.

For any auxiliary sequence $\left(h_{\varepsilon}\right)_{\mathcal{\varepsilon}>0} \subset H^{1}\left(0, T ; C^{0}(\bar{\omega}) \cap H_{0}^{1}(\omega)\right)$ with $h_{\varepsilon \mid t=0}=h_{0}^{\varepsilon}$ for all $\varepsilon>0$, we take a space-time regularization

$$
\delta_{\varepsilon}^{\star}:=N_{\varepsilon}(\delta) \in C_{\mathcal{C}}^{\infty}([0, T] \times \omega)
$$

of $\delta$ such that, if $h_{\varepsilon} \rightarrow \delta$ in $C^{0}([0, T] \times \bar{\omega})$ and $\partial_{t} h_{\varepsilon} \rightarrow \partial_{t} \delta$ in $L^{2}\left(0, T ; L^{2}(\omega)\right)$ as $\varepsilon \rightarrow 0$, then

$$
\begin{aligned}
N_{\varepsilon}\left(h_{\varepsilon}\right) & \rightarrow \delta \quad \text { in } C^{0}([0, T] \times \bar{\omega}) \\
\partial_{t} N_{\varepsilon}\left(h_{\varepsilon}\right) & \rightarrow \partial_{t} \delta \text { in } L^{2}\left(0, T ; L^{2}(\omega)\right) \\
N_{\varepsilon}(\delta)_{\mid t=0} & =h_{0}^{\varepsilon} .
\end{aligned}
$$

Note that we use the same parameter $\varepsilon$ introduced in the beginning of Step 1 for $h_{0}^{\varepsilon}, h_{1}^{\varepsilon}$, for $\delta_{\varepsilon}^{\star}$, the sequence $h_{\varepsilon}$, as well as for $N_{\varepsilon} ;$ moreover, $\delta$ depends on $\varepsilon$ via $h_{0}^{\varepsilon}$.

The operator $N_{\varepsilon}$ can be constructed as follows: Consider for each $\varepsilon>0$ a regularization $S_{\varepsilon}: H^{1}\left(0, T ; C^{0}(\bar{\omega}) \cap H_{0}^{1}(\omega)\right) \longrightarrow C^{\infty}\left([0, T] ; C_{0}^{\infty}(\omega)\right)$ such that $S_{\varepsilon}(z) \rightarrow z$ uniformly on 
bounded subsets of $H^{1}\left(0, T ; C^{0}(\bar{\omega}) \cap H_{0}^{1}(\omega)\right)$ as $\varepsilon \rightarrow 0$ and $\left(S_{\varepsilon}(z)\right)_{\mid t=0}=0$ if $z_{\mid t=0}=0$. Then, we set

$$
N_{\varepsilon}(\delta):=S_{\varepsilon}\left(\delta-\delta_{\mid t=0}\right)+h_{0}^{\varepsilon} \quad \forall \delta \in H^{1}\left(0, T ; C^{0}(\bar{\omega}) \cap H_{0}^{1}(\omega)\right) .
$$

Since $\left\|N_{\varepsilon}(\delta)-\delta\right\|_{C^{0}([0, T] \times \bar{\omega})}=\left\|S_{\varepsilon}\left(\delta-\delta_{\mid t=0}\right)-\left(\delta-h_{0}^{\varepsilon}\right)\right\|_{C^{0}([0, T] \times \bar{\omega})} \rightarrow 0$ as $\varepsilon \rightarrow 0$ and $M \geq 1+\delta \geq m$ on $[0, T] \times \bar{\omega}$, we can assume that

$$
2 M \geq 1+\delta_{\varepsilon}^{\star} \geq \frac{m}{2} \text { on }[0, T] \times \bar{\omega}
$$

by considering $\varepsilon>0$ small enough. For further technical details, we refer to Reference [18]. The properties (24) of $N_{\varepsilon}$ will be crucial in Step 6.3 below in the analysis of the fixed point operator $F_{\varepsilon}$, as in (52).

Furthermore, in order to regularize the non-linearity, we also introduce the space-time regularization $v_{\mathcal{\varepsilon}}^{\star}:=R_{\mathcal{\varepsilon}}(v) \in C^{\infty}\left((0, T) \times B_{2 M}\right)$ for $v \in L^{2}\left(0, T ; L^{2}\left(B_{2 M}\right)\right)$ such that

$$
R_{\varepsilon}\left(v_{\varepsilon}\right) \rightarrow v \text { in } L^{2}\left(0, T ; L^{2}\left(B_{2 M}\right)\right) \text { as } \varepsilon \rightarrow 0 \text { if } v_{\varepsilon} \rightarrow v \text { in } L^{2}\left(0, T ; L^{2}\left(B_{2 M}\right)\right) \text { as } \varepsilon \rightarrow 0 .
$$

For example, we can set $R_{\varepsilon}(v)=\varphi_{\varepsilon} * E_{0}(v)$ with $\left(\varphi_{\varepsilon}\right)_{\varepsilon>0} \subset C^{\infty}\left(\mathbb{R}^{4}\right)$ being an approximate identity with compact support in $(0, T) \times B_{2 M}$, and $E_{0}$ denotes the extension by zero on $\mathbb{R}^{4}$. Step 2: The approximate, almost linearized problem

Given $u_{0}^{\varepsilon}, h_{0}^{\varepsilon}, h_{1}^{\varepsilon}$ and $\delta_{\varepsilon}^{\star}=N_{\varepsilon}(\delta), v_{\varepsilon}^{\star}=R_{\varepsilon}(v)$ (for an arbitrary $v \in L^{2}\left(0, T ; L^{2}\left(B_{2 M}\right)\right)$ ), as in Step 1, we consider the following, almost linearized, approximate problem: Find $\left(u_{\varepsilon}, h_{\varepsilon}\right)$ such that

(i) $u_{\varepsilon} \in V_{\delta_{\varepsilon}^{\star}} \cap L^{\infty}\left(0, T ; L^{2}\left(\Omega_{\delta_{\varepsilon}^{\star}(t)}\right)\right)$,

(ii) $h_{\varepsilon} \in W^{1, \infty}\left(0, T ; L^{2}(\omega)\right) \cap H^{1}\left(0, T ; H_{0}^{2}(\omega)\right)$,

(iii) $u_{\varepsilon}\left(t, x, y, 1+\delta_{\varepsilon}^{\star}(t, x, y)\right)=\left(0,0, \partial_{t} h_{\varepsilon}(t, x, y)\right)^{\top}$ on $[0, T] \times \omega$,

(iv) $\partial_{t} u_{\varepsilon} \in L^{2}\left(0, T ; L^{2}\left(\Omega_{\delta_{\varepsilon}^{\star}(t)}\right)\right)$,

(v) $\partial_{t}^{2} h_{\varepsilon} \in L^{2}\left(0, T ; L^{2}(\omega)\right)$,

(vi) $u_{\varepsilon}(0)=u_{0}^{\varepsilon}, h_{\varepsilon}(0)=h_{0}^{\varepsilon}$ and $\partial_{t} h_{\varepsilon}(0)=h_{1}^{\varepsilon}$,

(vii) for all $\phi_{\varepsilon} \in V_{\delta_{\varepsilon}^{\star}}$ and $b \in L^{2}\left(0, T ; H_{0}^{2}(\omega)\right)$ such that $\phi_{\varepsilon}\left(t, x, y, 1+\delta_{\varepsilon}^{\star}(t, x, y)\right)$ $=(0,0, b(t, x, y))^{\top}$ on $[0, T] \times \omega$, we have

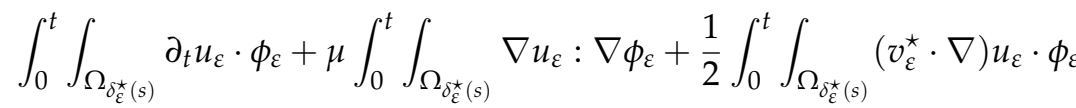

$$
\begin{aligned}
& -\frac{1}{2} \int_{0}^{t} \int_{\Omega_{\delta_{\varepsilon}^{\star}(s)}}\left(v_{\varepsilon}^{\star} \cdot \nabla\right) \phi_{\varepsilon} \cdot u_{\varepsilon}+\frac{1}{2} \int_{0}^{t} \int_{\omega} \partial_{t} h_{\varepsilon}\left(\partial_{t} \delta_{\varepsilon}^{\star}\right) b+\int_{0}^{t} \int_{\omega}\left(\partial_{t}^{2} h_{\varepsilon}\right) b \\
& +\int_{0}^{t} \int_{\omega} \Delta \partial_{t} h_{\varepsilon} \Delta b+\int_{0}^{t} \int_{\omega} \Delta h_{\varepsilon} \Delta b+\int_{0}^{t} \int_{\omega} \kappa G_{\varepsilon}\left(\partial_{t} h_{\varepsilon}\right) b=\int_{0}^{t} \int_{\Omega_{\delta_{\varepsilon}^{\star}(s)}} f \cdot \phi_{\varepsilon} .
\end{aligned}
$$

Here, we linearized the term $\int_{0}^{t} \int_{\omega}\left(\left|\partial_{t} h\right|-\left|\partial_{t} h-b\right|\right)$ with respect to $b$ via $\int_{0}^{t} \int_{\omega} G_{\varepsilon}\left(\partial_{t} h_{\varepsilon}\right) b$, with $G_{\varepsilon}$ being the derivative of $j_{\varepsilon}: \mathbb{R} \rightarrow \mathbb{R}, x \mapsto \sqrt{x^{2}+\varepsilon^{2}}$, i.e.,

$$
G_{\varepsilon}\left(\partial_{t} h_{\varepsilon}\right)=\frac{\partial_{t} h_{\varepsilon}}{\sqrt{\left|\partial_{t} h_{\varepsilon}\right|^{2}+\varepsilon^{2}}}
$$


However, $G_{\varepsilon}\left(\partial_{t} h_{\varepsilon}\right)$ is still non-linear in the unknown $h_{\mathcal{\varepsilon}}$. In addition, we wrote $\int_{\Omega_{\delta_{\varepsilon}^{\star}(s)}} \nabla u_{\varepsilon}$ : $\nabla \phi_{\varepsilon}$ as $\frac{1}{2} \int_{\Omega_{\delta_{\varepsilon}^{\star}(s)}} D u_{\varepsilon}: D \phi_{\varepsilon}$ which is possible due to Lemma 6. Furthermore, comparing (28) with (20), we reversed the integration by parts with respect to time. For $h_{\varepsilon}$, we used

$$
-\int_{0}^{t} \int_{\omega} \partial_{t} h_{\varepsilon} \partial_{t} b+\int_{\omega} \partial_{t} h_{\varepsilon}(t) b(t)-\int_{\omega} h_{1}^{\varepsilon} b(0)=\int_{0}^{t} \int_{\omega}\left(\partial_{t}^{2} h_{\varepsilon}\right) b
$$

which explains the new term $\int_{0}^{t} \int_{\omega}\left(\partial_{t}^{2} h_{\varepsilon}\right) b$ in (28). For $u_{\varepsilon}$, we used the Reynolds transport theorem to get

$$
\begin{aligned}
\int_{\Omega_{\delta_{\varepsilon}^{\star}(t)}} & u_{\varepsilon}(t) \phi(t)-\int_{0}^{t} \int_{\Omega_{\delta_{\varepsilon}^{\star}(s)}} u_{\varepsilon} \partial_{t} \phi_{\varepsilon}-\int_{\Omega_{h_{0}^{\varepsilon}}} u_{0}^{\varepsilon} \phi_{\varepsilon}(0) \\
= & \int_{0}^{t} \int_{\Omega_{\delta_{\varepsilon}^{\star}(s)}} \partial_{t} u_{\varepsilon} \cdot \phi_{\varepsilon}+2 \cdot \frac{1}{2} \int_{0}^{t} \int_{\omega} \partial_{t} h_{\varepsilon}\left(\partial_{t} \delta_{\varepsilon}^{\star}\right) b .
\end{aligned}
$$

This in combination with the replacement of the term $-\frac{1}{2} \int_{0}^{t} \int_{\omega}\left(\partial_{t} h\right)^{2} b$ in (20) by the more regular term $-\frac{1}{2} \int_{0}^{t} \int_{\omega} \partial_{t} h_{\varepsilon}\left(\partial_{t} \delta_{\varepsilon}^{\star}\right) b$ explains the term $\frac{1}{2} \int_{0}^{t} \int_{\omega} \partial_{t} h_{\varepsilon}\left(\partial_{t} \delta_{\varepsilon}^{\star}\right) b$ in (28).

Note that we are allowed to take the solution $\left(u_{\varepsilon}, \partial_{t} h_{\varepsilon}\right)$ as test function $(\phi, b)$ in $(28)$. This yields

$$
\begin{aligned}
& \quad \frac{1}{2}\left\|u_{\varepsilon}(t)\right\|_{\Omega_{\delta_{\varepsilon}^{\star}(t)}}^{2}+\mu \int_{0}^{t}\left\|\nabla u_{\varepsilon}\right\|_{L^{2}\left(\Omega_{\delta_{\varepsilon}^{\star}(s)}\right)}^{2}+\frac{1}{2}\left\|\partial_{t} h_{\varepsilon}(t)\right\|_{L^{2}(\omega)}^{2}+\int_{0}^{t}\left\|\Delta \partial_{t} h_{\mathcal{\varepsilon}}\right\|_{L^{2}(\omega)}^{2} \\
& +\frac{1}{2}\left\|\Delta h_{\varepsilon}(t)\right\|_{L^{2}(\omega)}^{2}+\int_{0}^{t} \int_{\omega} \kappa \frac{\left|\partial_{t} h_{\varepsilon}\right|^{2}}{\sqrt{\left|\partial_{t} h_{\varepsilon}\right|^{2}+\varepsilon^{2}}} \\
& =\int_{0}^{t} \int_{\Omega_{\delta_{\varepsilon}^{\star}(s)}} f \cdot u_{\varepsilon}+\frac{1}{2}\left\|u_{0}^{\varepsilon}\right\|_{L^{2}\left(\Omega_{h_{0}^{\varepsilon}}\right)}^{2}+\frac{1}{2}\left\|\Delta h_{0}\right\|_{L^{2}(\omega)}^{2}+\frac{1}{2}\left\|h_{1}\right\|_{L^{2}(\omega)}^{2} .
\end{aligned}
$$

Here, we used that

$$
\int_{\Omega_{\delta_{\varepsilon}^{\star}(s)}} \partial_{t} u_{\varepsilon} \cdot u_{\varepsilon}+\frac{1}{2} \int_{\omega}\left(\partial_{t} h_{\varepsilon}\right)^{2} \partial_{t} \delta_{\varepsilon}^{\star}=\frac{1}{2} \frac{d}{d t} \int_{\Omega_{\delta_{\varepsilon}^{\star}(s)}}\left|u_{\varepsilon}\right|^{2},
$$

which is true since the upper boundary of $\Omega_{\delta_{\varepsilon}^{\star}(t)}$ moves at the velocity $\left(0,0, \partial_{t} \delta_{\varepsilon}^{\star}\right)^{\top}$ and $u_{\varepsilon}\left(t, x, y, 1+\delta_{\varepsilon}^{\star}(t, x, y)\right)=\left(0,0, \partial_{t} h_{\varepsilon}(t, x, y)\right)^{\top}$ on $\omega$, see iii) above. Similar to the considerations in Section 2, we get

$$
\begin{array}{r}
\left\|u_{\mathcal{\varepsilon}}\right\|_{L^{\infty}\left(0, T ; L^{2}\left(\Omega_{\delta_{\varepsilon}^{\star}(t)}\right)\right)}+\left\|\nabla u_{\mathcal{\varepsilon}}\right\|_{L^{2}\left(0, t ; L^{2}\left(\Omega_{\delta_{\varepsilon}^{\star}(t)}\right)\right)}+\left\|\partial_{t} h_{\varepsilon}\right\|_{L^{\infty}\left(0, T ; H^{1}(\omega)\right)} \\
+\left\|\Delta h_{\mathcal{\varepsilon}}\right\|_{H^{1}\left(0, T ; L^{2}(\omega)\right)} \leq C^{\prime}
\end{array}
$$

with $C>0$ depending only on the data and $T$ but not on $\varepsilon>0, M$ or $m$. Due to $\omega$ being bounded, we also deduce by Poincaré's inequality, as in Lemma 7, that $u_{\varepsilon}$ is bounded in $L^{2}\left(0, T ; H^{1}\left(\Omega_{\delta_{\varepsilon}^{\star}(t)}\right)\right)$ by a constant independent of $\varepsilon>0$.

Now, we transform the domain $\Omega_{\delta_{\varepsilon}^{\star}(t)}$ to the reference configuration, the cylinder $Z=Z_{1}=\omega \times(0,1)$, via

$$
\chi_{\varepsilon}(t): Z \rightarrow \Omega_{\delta_{\varepsilon}^{\star}(t)}, \quad(x, y, z) \mapsto\left(x, y, z\left(1+\delta_{\varepsilon}^{\star}(t, x, y)\right)\right)^{\top} .
$$

Note that $\chi_{\varepsilon}(t)$ is a smooth diffeomorphism with $\operatorname{det} \nabla \chi_{\varepsilon}(t)=1+\delta_{\varepsilon}^{\star}(t)$ for all $t \in(0, T)$. For the sake of abbreviation, we set $\underline{\rho_{\varepsilon}}(t):=\rho \circ \chi_{\varepsilon}(t)$ for a function $\rho$ in the following. 
Then, the transformed system reads

$$
\begin{aligned}
& \int_{0}^{t} \int_{Z} \partial_{t} \underline{u_{\varepsilon}} \cdot \underline{\phi_{\varepsilon}}\left(1+\delta_{\varepsilon}^{\star}\right)-\int_{0}^{t} \int_{Z} \partial_{3} \underline{u_{\varepsilon}} \cdot \underline{\phi_{\varepsilon}} z \partial_{t} \delta_{\varepsilon}^{\star}+\mu \int_{0}^{t} \int_{Z}\left(\nabla \underline{u_{\varepsilon}} A_{\varepsilon}\right): \nabla \underline{\phi_{\varepsilon}} \\
& \quad+\frac{1}{2} \int_{0}^{t} \int_{Z}\left(\underline{v_{\varepsilon}^{\star}} \cdot\left(B_{\varepsilon} \nabla\right)\right) \underline{u_{\varepsilon}} \cdot \underline{\phi_{\varepsilon}}-\frac{1}{2} \int_{0}^{t} \int_{Z}\left(\underline{v_{\varepsilon}^{\star}} \cdot\left(B_{\varepsilon} \nabla\right)\right) \underline{\phi_{\varepsilon}} \cdot \underline{u_{\varepsilon}} \\
& \quad+\frac{1}{2} \int_{0}^{t} \int_{\omega} \partial_{t} h_{\varepsilon}\left(\partial_{t} \delta_{\varepsilon}^{\star}\right) b+\int_{0}^{t} \int_{\omega} \partial_{t}^{2} h_{\varepsilon} b+\int_{0}^{t} \int_{\omega} \Delta \partial_{t} h_{\varepsilon} \Delta b+\int_{0}^{t} \int_{\omega} \Delta h_{\varepsilon} \Delta b \\
& \quad+\int_{0}^{t} \int_{\omega} \kappa G_{\varepsilon}\left(\partial_{t} h_{\varepsilon}\right) b=\int_{0}^{t} \int_{Z} \underline{f} \cdot \underline{\phi_{\varepsilon}}\left(1+\delta_{\varepsilon}^{\star}\right)
\end{aligned}
$$

for all $\underline{\phi_{\varepsilon}} \in L^{2}\left(0, T ; H_{0, \Gamma_{0}}^{1}(Z), b \in L^{2}\left(0, T ; H_{0}^{2}(\omega)\right)\right.$ such that

$$
\underline{\phi_{\varepsilon}}(t, x, y, 1)=(0,0, b(t, x, y))^{\top} \text { on } \omega, \operatorname{div}\left(B_{\varepsilon}^{\top} \underline{\phi_{\varepsilon}}\right)=0 \text { in } Z .
$$

Here, $B_{\varepsilon}:=\left(\nabla \chi_{\varepsilon}\right)^{-\top}\left(1+\delta_{\varepsilon}^{\star}\right), A_{\varepsilon}:=\frac{1}{1+\delta_{\varepsilon}^{\star}} B_{\varepsilon}^{\top} B_{\varepsilon}$ and $\left(\underline{v_{\varepsilon}^{\star}} \cdot\left(B_{\varepsilon} \nabla\right)\right) \rho:=(\nabla \rho) B_{\varepsilon}^{\top} \underline{v_{\varepsilon}^{\star}}$. The interface condition transfers to

$$
\underline{u_{\mathcal{\varepsilon}}}(t, x, y, 1)=\left(0,0, \partial_{t} h_{\mathcal{\varepsilon}}(t, x, y)\right)^{\top}, \quad \forall(x, y) \in \omega .
$$

Step 3: The Galerkin procedure for the approximate, linearized problem

Now, we construct a Galerkin basis $\left(\psi_{i}^{0}\right)_{i \in \mathbb{N}}$ of the space $\left\{v \in H_{0}^{1}(Z) \mid \operatorname{div} v=0\right\}$ by the eigenfunctions of the Stokes problem, as in Reference [19] (Chapter 1, Section 2.6),

$$
\begin{aligned}
-\Delta \psi_{i}^{0}+\nabla p_{i}^{0} & =\mu_{i} \psi_{i}^{0} & & \text { in } Z, \\
\operatorname{div} \psi_{i}^{0} & =0 & & \text { in } Z, \\
\psi_{i}^{0} & =0 & & \text { on } \partial Z .
\end{aligned}
$$

Setting $\phi_{i}^{0, \varepsilon}=\phi_{i}^{0, \varepsilon}(t):=\left(B_{\mathcal{\varepsilon}}^{\top}(t)\right)^{-1} \psi_{i}^{0}$, we get for any $t \in[0, T]$ a basis of the space

$$
\left\{v \in H_{0}^{1}(Z) \mid \operatorname{div}\left(B_{\varepsilon}^{\top} v\right)=0 \text { in } Z\right\} .
$$

Let $\left(\xi_{j}\right)_{j \in \mathbb{N}}$ be a basis of $\left\{b \in H_{0}^{2}(\omega) \mid \int_{\omega} b=0\right\}$. Then, we construct $\left(\phi_{j}^{1, \varepsilon}\right)_{j \in \mathbb{N}}$ such that $\operatorname{div}\left(B_{\varepsilon}^{\top} \phi_{j}^{1, \varepsilon}\right)(t)=0$ and $\phi_{j}^{1, \varepsilon}(t, x, y, 1)=\left(0,0, \xi_{j}(x, y)\right)^{\top}$ for all $(x, y) \in \omega$. This can be achieved by solving the $t$-dependent modified Stokes problem

$$
\begin{aligned}
& -\Delta \phi_{j}^{1, \varepsilon}+\left(B_{\varepsilon} \cdot \nabla\right) p_{j}^{1, \varepsilon}=0 \quad \text { in } Z, \\
& \operatorname{div}\left(B_{\varepsilon}^{\top} \phi_{j}^{1, \varepsilon}\right)=0 \quad \text { in } Z \text {, } \\
& \phi_{j}^{1, \varepsilon}= \begin{cases}0 & \text { on } \Gamma_{0}, \\
\left(0,0, \xi_{j}\right)^{\top} & \text { on } \partial Z \backslash \Gamma_{0}\end{cases}
\end{aligned}
$$

in a weak sense. Furthermore,

$$
\left\|\phi_{j}^{1, \varepsilon}\right\|_{H^{1}(Z)}+\left\|p_{j}^{1, \varepsilon}\right\|_{L^{2}(Z)} \leq C_{\varepsilon}\left\|\xi_{j}\right\|_{H^{\frac{1}{2}}(\omega)} .
$$

Note that both $\phi_{i}^{0, \varepsilon}$ and $\phi_{i}^{1, \varepsilon}$ are smooth with respect to time since $B_{\varepsilon}$, via $\delta_{\varepsilon}^{*}$, has this property. Moreover, on $\partial Z$, all basis functions $\phi_{i}^{0, \varepsilon}, \phi_{i}^{1, \varepsilon}$ are independent of $t$, and even $\phi_{i}^{0, \varepsilon}=0$ on $\partial Z$. 
Now, we consider the $2 n$ equations

$$
\begin{aligned}
& \int_{Z} \partial_{t} \underline{u_{\varepsilon}^{n}} \cdot \phi_{i}^{0, \varepsilon}\left(1+\delta_{\varepsilon}^{\star}\right)-\int_{Z} \partial_{3} \underline{u_{\varepsilon}^{n}} \cdot \phi_{i}^{0, \varepsilon} z \partial_{t} \delta_{\varepsilon}^{\star}+\mu \int_{Z} \nabla \underline{u_{\varepsilon}^{n}} A_{\varepsilon}: \nabla \phi_{i}^{0, \varepsilon} \\
& \quad+\frac{1}{2} \int_{Z}\left(\underline{v_{\varepsilon}^{\star}} \cdot\left(B_{\varepsilon} \nabla\right)\right) \underline{u_{\varepsilon}^{n}} \cdot \phi_{i}^{0, \varepsilon}-\frac{1}{2} \int_{Z}\left(\underline{v_{\varepsilon}^{\star}} \cdot\left(B_{\varepsilon} \nabla\right)\right) \phi_{i}^{0, \varepsilon} \cdot \underline{u_{\varepsilon}^{n}}=\int_{Z} \underline{f} \cdot \phi_{i}^{0, \varepsilon}\left(1+\delta_{\varepsilon}^{\star}\right)
\end{aligned}
$$

for $i=1, \ldots, n$ and

$$
\begin{aligned}
& \int_{Z} \partial_{t} \underline{u_{\varepsilon}^{n}} \cdot \phi_{j}^{1, \varepsilon}\left(1+\delta_{\varepsilon}^{\star}\right)-\int_{Z} \partial_{3} \underline{u_{\varepsilon}^{n}} \cdot \phi_{j}^{1, \varepsilon} z \partial_{t} \delta_{\varepsilon}^{\star}+\mu \int_{Z} \nabla \underline{u_{\varepsilon}^{n}} A_{\varepsilon}: \nabla \phi_{j}^{1, \varepsilon} \\
& \quad+\frac{1}{2} \int_{Z}\left(\underline{v_{\varepsilon}^{\star}} \cdot\left(B_{\varepsilon} \nabla\right)\right) \underline{u_{\varepsilon}^{n}} \cdot \phi_{j}^{1, \varepsilon}-\frac{1}{2} \int_{Z}\left(\underline{v_{\varepsilon}^{\star}} \cdot\left(B_{\varepsilon} \nabla\right)\right) \phi_{j}^{1, \varepsilon} \cdot \underline{u_{\varepsilon}^{n}} \\
& +\frac{1}{2} \int_{\omega} \partial_{t} h_{\varepsilon}^{n}\left(\partial_{t} \delta_{\varepsilon}^{\star}\right) \xi_{j}+\int_{\omega}\left(\partial_{t}^{2} h_{\varepsilon}^{n}\right) \xi_{j}+\int_{\omega}\left(\Delta \partial_{t} h_{\varepsilon}^{n}\right) \Delta \xi_{j}+\int_{\omega} \Delta h_{\varepsilon}^{n} \Delta \xi_{j} \\
& \quad+\int_{\omega} \kappa G_{\varepsilon}\left(\partial_{t} h_{\varepsilon}^{n}\right) \xi_{j}=\int_{Z} \underline{f} \cdot \phi_{j}^{1, \varepsilon}\left(1+\delta_{\varepsilon}^{\star}\right)
\end{aligned}
$$

for $j=1, \ldots, n$, combined with the initial conditions

$$
h_{\varepsilon}^{n}(0)=h_{0}^{\varepsilon}, \underline{u_{\varepsilon}^{n}}(0)=\underline{u_{\varepsilon, 0}^{n}}, \quad \partial_{t} h_{\varepsilon}^{n}(0)=h_{\varepsilon, 1}^{n} ;
$$

here, $\underline{u_{\varepsilon, 0}^{n}}$ and $h_{\varepsilon, 1}^{n}$ denote the orthogonal projections of $\underline{u_{0}}{ }^{\varepsilon}$ and $h_{1}^{\varepsilon}$ onto the finite dimensional $\operatorname{spaces} \operatorname{span}\left(\phi_{i}^{0, \varepsilon}, \phi_{j}^{1, \varepsilon}\right)_{i, j \in\{1, \ldots, n\}}$ and $\operatorname{span}\left(\xi_{j}\right)_{j \in\{1, \ldots, n\}}$, respectively. Due to the smoothness of the involved functions with respect to time, we get the existence of a solution $\left(\underline{u_{\varepsilon}^{n}}, h_{\varepsilon}^{n}\right)$ of the form

$$
h_{\varepsilon}^{n}(t)=\sum_{j=1}^{n} \beta_{j}(t) \xi_{j}+h_{0}^{\varepsilon} \quad \text { and } \quad \underline{u_{\varepsilon}^{n}}(t)=\sum_{i=1}^{n} \alpha_{i}(t) \phi_{i}^{0, \varepsilon}+\sum_{j=1}^{n} \dot{\beta}_{j}(t) \phi_{j}^{1, \varepsilon}
$$

on $[0, T]$. In order to prove the unique existence of the solution, we first plug (37) into (35) and (36) to have a system that we want to solve for the unknowns $\alpha:=\left(\alpha_{1}, \ldots, \alpha_{n}\right)$ and $\beta:=\left(\beta_{1}, \ldots, \beta_{n}\right)$. This, in turn, can be done by reducing it to a system of first order with respect to time. This first order system for $(\beta, \alpha, \dot{\beta})^{\top}$ (without initial conditions) reads

$$
\left(\begin{array}{cc}
I_{n \times n} & 0_{n \times 2 n} \\
0_{2 n \times n} & M_{\mathcal{\varepsilon}}(t)+N
\end{array}\right) \partial_{t}\left(\begin{array}{c}
\beta \\
\alpha \\
\dot{\beta}
\end{array}\right)=\left(\begin{array}{c}
\dot{\beta} \\
F_{\varepsilon, t}(\beta, \alpha, \dot{\beta})
\end{array}\right) .
$$

Here, $F_{\varepsilon, t}$ denotes all lower-order terms that do not involve $\dot{\alpha}$ or $\ddot{\beta}$. Since the equation is linear, so is $F_{\varepsilon, t} \in \mathbb{R}^{2 n \times 3 n}$. Furthermore, $F_{\varepsilon, t}$ is smooth with respect to time. Obviously, $M_{\varepsilon}(t)+N \in \mathbb{R}^{2 n \times 2 n}$ is given by

$$
\begin{aligned}
& M_{\mathcal{\varepsilon}}(t)+N= \\
& \left(\begin{array}{cc}
\left(\int_{Z} \phi_{i}^{0, \varepsilon} \cdot \phi_{j}^{0, \varepsilon}\left(1+\delta_{\varepsilon}^{\star}\right)\right)_{i, j} & \left(\int_{Z} \phi_{i}^{0, \varepsilon} \cdot \phi_{j}^{1, \varepsilon}\left(1+\delta_{\varepsilon}^{\star}\right)\right)_{i, j} \\
\left(\int_{Z} \phi_{j}^{0, \varepsilon} \cdot \phi_{i}^{1, \varepsilon}\left(1+\delta_{\varepsilon}^{\star}\right)\right)_{i, j} & \left(\int_{Z} \phi_{i}^{1, \varepsilon} \cdot \phi_{j}^{1, \varepsilon}\left(1+\delta_{\varepsilon}^{\star}\right)\right)_{i, j}
\end{array}\right)+\left(\begin{array}{cc}
0 & 0 \\
0 & \left(\int_{\omega} \xi_{i} \xi_{j}\right)_{i, j}
\end{array}\right),
\end{aligned}
$$

where $i, j \in\{1, \ldots, n\}$. Obviously, $M_{\mathcal{E}}(t)$ is smooth with respect to time, too, and it is easy to check that $M_{\varepsilon}(t)+N$ is positive definite and, hence, invertible. Therefore, we deduce the existence of a unique solution of the form as in (37) that satisfies the mentioned initial conditions. 
Now, we multiply equation (35) with $\alpha_{i}$ and sum over $i \in\{1, \ldots, n\}$. By analogy, we multiply (36) with $\dot{\beta}_{j}$ and sum over $j \in\{1, \ldots, n\}$. Adding those two terms yields

$$
\begin{aligned}
& \int_{Z} \partial_{t} \underline{u_{\varepsilon}^{n}} \cdot \underline{u_{\varepsilon}^{n}}\left(1+\delta_{\varepsilon}^{\star}\right)-\int_{Z} \partial_{3} \underline{u_{\varepsilon}^{n}} \cdot \underline{u_{\varepsilon}^{n}} z \partial_{t} \delta_{\varepsilon}^{\star} \\
& +\mu \int_{Z} \nabla \underline{u_{\varepsilon}^{n}} A_{\varepsilon}: \nabla \underline{u_{\varepsilon}^{n}}+\frac{1}{2} \int_{\omega} \partial_{t} h_{\varepsilon}^{n}\left(\partial_{t} \delta_{\varepsilon}^{\star}\right) \partial_{t} h_{\varepsilon}^{n}+\int_{\omega}\left(\partial_{t}^{2} h_{\varepsilon}^{n}\right) \partial_{t} h_{\varepsilon}^{n} \\
& +\int_{\omega}\left(\Delta \partial_{t} h_{\varepsilon}^{n}\right) \Delta \partial_{t} h_{\varepsilon}^{n}+\int_{\omega} \Delta h_{\varepsilon}^{n} \Delta \partial_{t} h_{\varepsilon}^{n}+\int_{\omega} \kappa G_{\varepsilon}\left(\partial_{t} h_{\varepsilon}^{n}\right) \partial_{t} h_{\varepsilon}^{n}=\int_{Z} \underline{f} \cdot \underline{u_{\varepsilon}^{n}}\left(1+\delta_{\varepsilon}^{\star}\right) .
\end{aligned}
$$

Since $\partial_{t}\left(1+\delta_{\varepsilon}^{\star}\right)=\operatorname{div}\left(0,0, z \partial_{t} \delta_{\varepsilon}^{\star}\right)^{\top}$, we get, with the exterior normal vector $n_{Z}$ of $\omega \times$ $(0,1)$, that

$$
\begin{aligned}
& \frac{1}{2} \int_{Z} \frac{\left|u_{\varepsilon}^{n}\right|^{2} \partial_{t}\left(1+\delta_{\varepsilon}^{\star}\right)=\left.\frac{1}{2} \int_{Z} \underline{\mid u_{\varepsilon}^{n}}\right|^{2} \operatorname{div}\left(0,0, z \partial_{t} \delta_{\varepsilon}^{\star}\right)^{\top}}{\quad=\frac{1}{2} \int_{\partial Z \backslash \Gamma_{0}} \underline{\left|u_{\varepsilon}^{n}\right|^{2}}\left(0,0, z \partial_{t} \delta_{\varepsilon}^{\star}\right)^{\top} \cdot n_{Z}-\int_{Z} \partial_{3} \underline{u_{\varepsilon}^{n}} \cdot \underline{u_{\varepsilon}^{n}} z \partial_{t} \delta_{\varepsilon}^{\star}} \\
& \quad=\frac{1}{2} \int_{\omega}\left(\partial_{t} h_{\varepsilon}^{n}\right)^{2} \partial_{t} \delta_{\varepsilon}^{\star}-\int_{Z} \partial_{3} \underline{u_{\varepsilon}^{n}} \cdot \underline{u_{\varepsilon}^{n}} z \partial_{t} \delta_{\varepsilon}^{\star} .
\end{aligned}
$$

Therefore, we deduce

$$
\left.\frac{1}{2} \frac{d}{d t} \int_{Z} \underline{\mid u_{\varepsilon}^{n}}\right|^{2}\left(1+\delta_{\varepsilon}^{\star}\right)=\int_{Z} \partial_{t} \underline{u_{\varepsilon}^{n}} \cdot \underline{u_{\varepsilon}^{n}}\left(1+\delta_{\varepsilon}^{\star}\right)+\frac{1}{2} \int_{\omega}\left(\partial_{t} h_{\varepsilon}^{n}\right)^{2} \partial_{t} \delta_{\varepsilon}^{\star}-\int_{Z} \partial_{3} \underline{u_{\varepsilon}^{n}} \cdot \underline{u_{\varepsilon}^{n}} z \partial_{t} \delta_{\varepsilon}^{\star} .
$$

Plugging this into (38), we get

$$
\begin{aligned}
& \frac{1}{2} \frac{d}{d t} \int_{Z}\left|\underline{u_{\varepsilon}^{n}}\right|^{2}\left(1+\delta_{\varepsilon}^{\star}\right)+\mu \int_{Z} \nabla \underline{u_{\varepsilon}^{n}} A_{\varepsilon}: \nabla \underline{u_{\varepsilon}^{n}}+\int_{\omega}\left(\partial_{t}^{2} h_{\varepsilon}^{n}\right) \partial_{t} h_{\varepsilon}^{n} \\
& \quad+\int_{\omega}\left(\Delta \partial_{t} h_{\varepsilon}^{n}\right)^{2}+\int_{\omega} \Delta h_{\varepsilon}^{n} \Delta \partial_{t} h_{\varepsilon}^{n}+\int_{\omega} \kappa G_{\varepsilon}\left(\partial_{t} h_{\varepsilon}^{n}\right) \partial_{t} h_{\varepsilon}^{n}=\int_{Z} \underline{f} \cdot \underline{u_{\varepsilon}^{n}}\left(1+\delta_{\varepsilon}^{\star}\right) .
\end{aligned}
$$

Similarly as done in Section 2, along with $G_{\varepsilon}\left(\partial_{t} h_{\varepsilon}^{n}\right) \partial_{t} h_{\varepsilon}^{n} \geq 0$ and using that $A_{\varepsilon}$ is elliptic with a constant independent of $n$, we can integrate in time and apply Grönwall's inequality to conclude that

$$
\begin{aligned}
& \left\|\underline{u_{\varepsilon}^{n}}\right\|_{L^{\infty}\left(0, T ; L^{2}(Z)\right)}+\left\|\nabla \underline{u_{\varepsilon}^{n}}\right\|_{L^{2}\left(0, T ; L^{2}(Z)\right)}+\left\|\partial_{t} h_{\varepsilon}^{n}\right\|_{L^{\infty}\left(0, T ; L^{2}(\omega)\right)}+\left\|\Delta h_{\varepsilon}^{n}\right\|_{H^{1}\left(0, T ; L^{2}(\omega)\right)} \\
& \quad \leq C\left(T,\left\|u_{0}\right\|_{L^{2}\left(\Omega_{h_{0}}\right)},\|f\|_{L^{2}\left((0, T) \times \mathbb{R}^{3}\right)},\left\|h_{0}\right\|_{H_{0}^{2}(\omega)},\left\|h_{1}\right\|_{L^{2}(\omega)}, \varepsilon, m, M\right) .
\end{aligned}
$$

Here, the constant $C>0$ depends on $\varepsilon, m$ and $M$. However, if we return to the problem in the deformed configuration, we obtain-similarly as done in Section 2-that

$$
\begin{aligned}
& \left\|u_{\varepsilon}^{n}\right\|_{L^{\infty}\left(0, T ; L^{2}\left(\Omega_{\delta_{\varepsilon}^{\star}(t)}\right)\right.}+\left\|\nabla u_{\varepsilon}^{n}\right\|_{L^{2}\left(0, T ; L^{2}\left(\Omega_{\delta_{\varepsilon}^{\star}(t)}\right)\right.}+\left\|\partial_{t} h_{\varepsilon}^{n}\right\|_{L^{\infty}\left(0, T ; L^{2}(\omega)\right)} \\
& \quad+\left\|\Delta h_{\varepsilon}^{n}\right\|_{H^{1}\left(0, T ; L^{2}(\omega)\right)} \leq C\left(T,\left\|u_{0}\right\|_{L^{2}\left(\Omega_{h_{0}}\right)},\|f\|_{L^{2}\left((0, T) \times \mathbb{R}^{3}\right)},\left\|h_{0}\right\|_{H_{0}^{2}(\omega)},\left\|h_{1}\right\|_{L^{2}(\omega)}\right)^{\prime}
\end{aligned}
$$

with $C>0$ independent of $\varepsilon, m$, and $M$.

Step 4: Uniform estimates of time derivatives in $n$

In order to obtain a solution for $n \rightarrow \infty$, we still need additional estimates of the largest time derivatives. To be more specific, we need the estimate

$$
\left\|\partial_{t} \underline{u_{\varepsilon}^{n}}\right\|_{L^{2}\left(0, T ; L^{2}(Z)\right)}+\left\|\partial_{t t} h_{\varepsilon}^{n}\right\|_{L^{2}\left(0, T ; L^{2}(\omega)\right)} \leq \tilde{C}(\varepsilon),
$$

with $\tilde{C}(\varepsilon)>0$ independent of $n$ but possibly dependent on the given data, $m, M$, and $\varepsilon$. 
In order to achieve this estimate, we multiply (35) with $\dot{\alpha}_{i}$ and sum over $i \in\{1, \ldots, n\}$. In a similar fashion, we multiply (36) with $\ddot{\beta}_{j}$ and sum over $j \in\{1, \ldots, n\}$. For simplicity, we omit the indices $\varepsilon$ and $n$ in the following and obtain, after adding the two sums,

$$
\begin{aligned}
\int_{Z} & \left|\sum_{i=1}^{2 n} \dot{\lambda}_{i} \phi_{i}\right|^{2}\left(1+\delta^{\star}\right)-\int_{Z} \partial_{3} \underline{u} \cdot \sum_{i=1}^{2 n} \dot{\lambda}_{i} \phi_{i} z \partial_{t} \delta^{\star} \\
& +\int_{Z} \sum_{i=1}^{2 n} \dot{\lambda}_{i} \phi_{i} \cdot \sum_{i=1}^{2 n} \lambda_{i} \partial_{t} \phi_{i}\left(1+\delta^{\star}\right)+\mu \int_{Z} \nabla \underline{u} A: \nabla\left(\sum_{i=1}^{2 n} \dot{\lambda}_{i} \phi_{i}\right) \\
& +\frac{1}{2} \int_{Z}\left(v^{\star} \cdot(B \nabla)\right) \underline{u} \cdot \sum_{i=1}^{2 n} \dot{\lambda}_{i} \phi_{i}-\frac{1}{2} \int_{Z}\left(v^{\star} \cdot(B \nabla)\right) \sum_{i=1}^{2 n} \dot{\lambda}_{i} \phi_{i} \cdot \underline{u} \\
& +\frac{1}{2} \int_{\omega} \partial_{t} h \partial_{t} \delta^{\star} \partial_{t}^{2} h+\int_{\omega}\left(\partial_{t}^{2} h\right)^{2}+\int_{\omega} \Delta \partial_{t} h \Delta \partial_{t}^{2} h+\int_{\omega} \Delta h \Delta \partial_{t}^{2} h+\int_{\omega} \kappa G\left(\partial_{t} h\right) \partial_{t}^{2} h \\
= & \int_{Z} \underline{f} \cdot \sum_{i=1}^{2 n} \dot{\lambda}_{i} \phi_{i}\left(1+\delta^{\star}\right)
\end{aligned}
$$

with

$$
\lambda_{i}:=\left\{\begin{array}{ll}
\alpha_{i,} & i \in\{1, \ldots, n\}, \\
\dot{\beta}_{i-n}, & i \in\{n+1, \ldots, 2 n\},
\end{array} \quad \text { and } \quad \phi_{i}:= \begin{cases}\phi_{i}^{0}, & i \in\{1, \ldots, n\}, \\
\phi_{i-n^{\prime}}^{1} & i \in\{n+1, \ldots, 2 n\} .\end{cases}\right.
$$

Hence, we get that

$$
\begin{aligned}
&\left\|\sum_{i=1}^{2 n} \dot{\lambda}_{i} \phi_{i} \sqrt{1+\delta^{\star}}\right\|_{L^{2}(Z)}^{2}+\frac{\mu}{2} \frac{d}{d t} \int_{Z} \nabla \underline{u} A: \nabla \underline{u}+\left\|\partial_{t}^{2} h\right\|_{L^{2}(\omega)}^{2}+\frac{1}{2} \frac{d}{d t} \int_{\omega}\left(\Delta \partial_{t} h\right)^{2} \\
&=- \int_{Z} \sum_{i=1}^{2 n} \dot{\lambda}_{i} \phi_{i} \sum_{i=1}^{2 n} \lambda_{i} \partial_{t} \phi_{i}\left(1+\delta^{\star}\right)+\frac{\mu}{2} \int_{Z} \nabla \underline{u} \partial_{t} A: \nabla \underline{u} \\
&+\mu \int_{Z} \nabla \underline{u} A: \nabla \sum_{i=1}^{2 n} \lambda_{i} \partial_{t} \phi_{i}-\frac{1}{2} \int_{Z}\left(v^{\star} \cdot(B \nabla)\right) \underline{u} \cdot \sum_{i=1}^{2 n} \dot{\lambda}_{i} \phi_{i} \\
&+\frac{1}{2} \int_{Z}\left(v^{\star} \cdot(B \nabla)\right) \sum_{i=1}^{2 n} \dot{\lambda}_{i} \phi_{i} \cdot \underline{u}+\int_{Z} \partial_{3} \underline{u} \cdot \sum_{i=1}^{2 n} \dot{\lambda}_{i} \phi_{i} z \partial_{t} \delta^{\star}-\frac{1}{2} \int_{\omega} \partial_{t} h \partial_{t} \delta^{\star} \partial_{t}^{2} h \\
&+\int_{\omega}\left(\Delta \partial_{t} h\right)^{2}-\frac{d}{d t} \int_{\omega} \Delta h \Delta \partial_{t} h-\int_{\omega} \kappa G\left(\partial_{t} h\right) \partial_{t}^{2} h+\int_{Z} \underline{f} \cdot \sum_{i=1}^{2 n} \dot{\lambda}_{i} \phi_{i}\left(1+\delta^{\star}\right) .
\end{aligned}
$$


Then, an integration in time implies that

$$
\begin{aligned}
\int_{0}^{t} & \sum_{i=1}^{2 n} \dot{\lambda}_{i} \phi_{i} \sqrt{1+\delta^{\star}} \|_{L^{2}(Z)}^{2}+\frac{\mu}{2} \int_{Z} \nabla \underline{u}(t) A(t): \nabla \underline{u}(t) \\
& +\int_{0}^{t}\left\|\partial_{t}^{2} h\right\|_{L^{2}(\omega)}^{2}+\frac{1}{2}\left\|\Delta \partial_{t} h(t)\right\|_{L^{2}(\omega)}^{2} \\
= & \frac{\mu}{2} \int_{Z} \nabla \underline{u}(0) A(0): \nabla \underline{u}(0)+\frac{1}{2}\left\|\Delta \partial_{t} h(0)\right\|_{L^{2}(\omega)}^{2}+\int_{\omega} \Delta h(0) \Delta \partial_{t} h(0) \\
& -\int_{0}^{t} \int_{Z} \sum_{i=1}^{2 n} \dot{\lambda}_{i} \phi_{i} \sum_{i=1}^{2 n} \lambda_{i} \partial_{t} \phi_{i}\left(1+\delta^{\star}\right)+\frac{\mu}{2} \int_{0}^{t} \int_{Z} \nabla \underline{u} \partial_{t} A: \nabla \underline{u} \\
& +\mu \int_{0}^{t} \int_{Z} \nabla \underline{u} A: \nabla \sum_{i=1}^{2 n} \lambda_{i} \partial_{t} \phi_{i}-\frac{1}{2} \int_{0}^{t} \int_{Z}\left(\underline{v}^{\star} \cdot(B \nabla)\right) \underline{u} \cdot \sum_{i=1}^{2 n} \dot{\lambda}_{i} \phi_{i}+\ldots \\
& \ldots+\frac{1}{2} \int_{0}^{t} \int_{Z}\left(\underline{v}^{\star} \cdot(B \nabla)\right) \sum_{i=1}^{2 n} \dot{\lambda}_{i} \phi_{i} \cdot \underline{u}+\int_{0}^{t} \int_{Z} \partial_{3} \underline{u} \cdot \sum_{i=1}^{2 n} \dot{\lambda}_{i} \phi_{i} z \partial_{t} \delta^{\star} \\
& -\frac{1}{2} \int_{0}^{t} \int_{\omega} \partial_{t} h \partial_{t} \delta^{\star} \partial_{t}^{2} h+\int_{0}^{t}\left\|\Delta \partial_{t} h\right\|_{L^{2}(\omega)}^{2}-\int_{\omega} \Delta h(t) \Delta \partial_{t} h(t)-\int_{0}^{t} \int_{\omega} \kappa G\left(\partial_{t} h\right) \partial_{t}^{2} h \\
& +\int_{0}^{t} \int_{Z} \underline{f} \cdot \sum_{i=1}^{2 n} \dot{\lambda}_{i} \phi_{i}\left(1+\delta^{\star}\right) .
\end{aligned}
$$

Now, we have to estimate every term on the right-hand side of (41) by a constant independent of $n$, but possibly depending on $\varepsilon, m, M$, and on $\delta$ and $v$. The first three pose no problem since they depend on the initial data that are smooth. For the fourth term, Hölder's and Young's inequality imply that

$$
\begin{aligned}
& \left|\int_{0}^{t} \int_{Z} \sum_{i=1}^{2 n} \dot{\lambda}_{i} \phi_{i} \sum_{i=1}^{2 n} \lambda_{i} \partial_{t} \phi_{i}\left(1+\delta^{\star}\right)\right| \\
& \quad \leq \frac{1}{8}\left\|\sum_{i=1}^{2 n} \dot{\lambda}_{i} \phi_{i} \sqrt{1+\delta^{\star}}\right\|_{L^{2}\left(0, t ; L^{2}(Z)\right)}^{2}+C\left\|\sum_{i=1}^{2 n} \lambda_{i} \partial_{t} \phi_{i}\right\|_{L^{2}\left(0, t ; L^{2}(Z)\right)}^{2} .
\end{aligned}
$$

Let us estimate the second summand of the right-hand side of (42). Remembering that $\phi_{i}^{0, \varepsilon}=\left(B_{\varepsilon}\right)^{-\top} \psi_{i}^{0}$, we get that

$$
\sum_{i=1}^{2 n} \lambda_{i} \partial_{t} \phi_{i}=\sum_{i=1}^{n} \alpha_{i} \partial_{t}\left(B^{-\top} \psi_{i}^{0}\right)+\sum_{j=1}^{n} \dot{\beta}_{j} \partial_{t} \phi_{j}^{1}=\sum_{i=1}^{n} \alpha_{i}\left(\partial_{t} B^{-\top}\right) B^{\top} \phi_{i}^{0}+\sum_{j=1}^{n} \dot{\beta}_{j} \partial_{t} \phi_{j}^{1} .
$$

Concerning the second term, we recall that $\phi_{j}^{1}$ solves the Stokes-like problem (33), and, hence, $\sum_{j=1}^{n} \dot{\beta}_{j} \partial_{t} \phi_{j}^{1}$ solves

$$
\begin{array}{rlrl}
-\Delta\left(\sum_{j=1}^{n} \dot{\beta}_{j} \partial_{t} \phi_{j}^{1}\right)+(B \nabla)\left(\sum_{j=1}^{n} \dot{\beta}_{j} \partial_{t} p_{j}^{1}\right) & =-\left(\partial_{t} B \nabla\right) \sum_{j=1}^{n} \dot{\beta}_{j} p_{j}^{1} & & \text { in } Z \\
\operatorname{div}\left(B^{\top} \sum_{j=1}^{n} \dot{\beta}_{j} \partial_{t} \phi_{j}^{1}\right) & =-\operatorname{div}\left(\partial_{t} B^{\top} \sum_{j=1}^{n} \dot{\beta}_{j} \phi_{j}^{1}\right) & \text { in } Z \\
\sum_{j=1}^{n} \dot{\beta}_{j} \partial_{t} \phi_{j}^{1} & =0 & & \text { on } \partial Z
\end{array}
$$


in the weak sense. Due to the duality estimate

$$
\begin{aligned}
\left|\left\langle-\left(\partial_{t} B \nabla\right) \sum_{j=1}^{n} \dot{\beta}_{j} p_{j}^{1}, \phi\right\rangle_{\left(H_{0}^{1}\right)^{\prime}, H_{0}^{1}}\right| & =\left|\int_{Z} \sum_{j=1}^{n} \dot{\beta}_{j} p_{j}^{1} \operatorname{div}\left(\left(\partial_{t} B^{\top}\right) \phi\right)\right| \\
& \leq C_{\varepsilon}\left\|\sum_{j=1}^{n} \dot{\beta}_{j} p_{j}^{1}\right\|_{L^{2}(Z)}\|\phi\|_{H^{1}(Z)}
\end{aligned}
$$

for all test functions $\phi \in H_{0}^{1}(Z)$, we get that

$$
\left\|\sum_{j=1}^{n} \dot{\beta}_{j} \partial_{t} \phi_{j}^{1}\right\|_{H^{1}(Z)} \leq C\left(\left\|\sum_{j=1}^{n} \dot{\beta}_{j} p_{j}^{1}\right\|_{L^{2}(Z)}+\left\|\sum_{j=1}^{n} \dot{\beta}_{j} \phi_{j}^{1}\right\|_{H^{1}(Z)}\right) .
$$

Now, using the equation solved by $\left(\phi_{j}^{1}, p_{j}\right)$ and their continuous dependence on the given data, as in (34), we can estimate further:

$$
\left\|\sum_{j=1}^{n} \dot{\beta}_{j} p_{j}^{1}\right\|_{L^{2}(Z)}+\left\|\sum_{j=1}^{n} \dot{\beta}_{j} \phi_{j}^{1}\right\|_{H^{1}(Z)} \leq C\left\|\sum_{j=1}^{n} \dot{\beta}_{j} \xi_{j}\right\|_{H^{1 / 2}(\omega)}=C\left\|\partial_{t} h\right\|_{H^{1 / 2}(\omega)} .
$$

Combining the last two estimates, we have

$$
\left\|\sum_{j=1}^{n} \dot{\beta}_{j} \partial_{t} \phi_{j}^{1}\right\|_{L^{2}\left(0, t ; H^{1}(Z)\right)} \leq C\left\|\partial_{t} h\right\|_{L^{2}\left(0, t ; H^{1 / 2}(\omega)\right)} \leq C\left\|\partial_{t} h\right\|_{L^{2}\left(0, t ; H_{0}^{2}(\omega)\right)} \stackrel{(39)}{\leq} C .
$$

The term $\left(\partial_{t} B^{-\top}\right) B^{\top}$ in (43) is uniformly bounded on $(0, T) \times Z$ due to the smoothness and boundedness of $\delta^{\star}$. The bound may depend on $\varepsilon$ though. Hence, we get that

$$
\begin{array}{r}
\left\|\sum_{i=1}^{n} \alpha_{i}\left(\partial_{t} B^{-\top}\right) B^{\top} \phi_{i}^{0}\right\|_{L^{2}\left(0, t ; H^{1}(Z)\right)} \leq C\left\|\sum_{i=1}^{n} \alpha_{i} \phi_{i}^{0}\right\|_{L^{2}\left(0, t ; H^{1}(Z)\right)} \\
\leq C\|\underline{u}\|_{L^{2}\left(0, t ; H^{1}(Z)\right)}+C\left\|\sum_{j=1}^{n} \dot{\beta}_{j}(t) \phi_{j}^{1}\right\|_{L^{2}\left(0, t ; H^{1}(Z)\right)} \leq C,
\end{array}
$$

where we use the estimate (44) for the term involving $\dot{\beta}$ and (39) for the term involving $\underline{u}$. Therefore, we get from (42) that

$$
\left|\int_{0}^{t} \int_{Z} \sum_{i=1}^{2 n} \dot{\lambda}_{i} \phi_{i} \sum_{i=1}^{2 n} \lambda_{i} \partial_{t} \phi_{i}\left(1+\delta^{\star}\right)\right| \leq \frac{1}{8}\left\|\sum_{i=1}^{2 n} \dot{\lambda}_{i} \phi_{i}\right\|_{L^{2}\left(0, t ; L^{2}(Z)\right)}^{2}+C .
$$

The integral

$$
\frac{\mu}{2} \int_{0}^{t} \int_{Z} \nabla \underline{u} \partial_{t} A: \nabla \underline{u}
$$

is bounded independently of $n$ since $\underline{u}$ is bounded uniformly in $L^{2}\left(0, t ; H^{1}(Z)\right)$, as in (39), and $\partial_{t} A$ is bounded in $L^{\infty}((0, t) \times \omega \times(0,1))$ due to $\delta^{\star}$ being independent of $n$. Concerning the term

$$
\mu \int_{0}^{t} \int_{Z} \nabla \underline{u} A: \nabla\left(\sum_{i=1}^{2 n} \lambda_{i} \partial_{t} \phi_{i}\right)
$$


we use again (43) and deduce that

$$
\begin{aligned}
\| \sum_{i=1}^{2 n} \lambda_{i} \partial_{t} \phi_{i} & \left\|_{L^{2}\left(0, t ; H^{1}(Z)\right)} \leq\right\| \sum_{i=1}^{n} \alpha_{i} \partial_{t} \phi_{i}^{0}\left\|_{L^{2}\left(0, t ; H^{1}(Z)\right)}+\right\| \sum_{j=1}^{n} \dot{\beta}_{j} \partial_{t} \phi_{j}^{1} \|_{L^{2}\left(0, t ; H^{1}(Z)\right)} \\
& =\left\|\sum_{i=1}^{n} \alpha_{i}\left(\partial_{t} B^{-T}\right) B^{T} \phi_{i}^{0}\right\|_{L^{2}\left(0, t ; H^{1}(Z)\right)}+\left\|\sum_{j=1}^{n} \dot{\beta}_{j} \partial_{t} \phi_{j}^{1}\right\|_{L^{2}\left(0, t ; H^{1}(Z)\right)}
\end{aligned}
$$

is bounded due to (46) and (45).

For the next term in (41) involving $v^{\star}=R_{\varepsilon}(v)=\varphi_{\varepsilon} * E_{0}(v)$, as in (27), we use

$$
\left\|{\underline{v^{\star}}}_{L^{\infty}((0, t) \times Z)} \leq\right\| \varphi_{\varepsilon} * E_{0}(v)\left\|_{L^{\infty}\left((0, t) \times \mathbb{R}^{3}\right)} \leq C_{\varepsilon}\right\| v \|_{L^{2}\left((0, t) \times B_{2 M}\right)},
$$

and the boundedness of $\underline{u}$ in $L^{2}\left(0, t ; H^{1}(Z)\right)$, to get that

$$
\begin{aligned}
& \left|\frac{1}{2} \int_{0}^{t} \int_{Z}\left(\underline{v}^{\star} \cdot(B \nabla)\right) \underline{u} \cdot \sum_{i=1}^{2 n} \dot{\lambda}_{i} \phi_{i}\right| \\
& \quad \leq C\|v\|_{L^{2}\left((0, t) \times B_{2 M}\right)}^{2}+\frac{1}{8}\left\|\sum_{i=1}^{2 n} \dot{\lambda}_{i} \phi_{i} \sqrt{1+\delta^{\star}}\right\|_{L^{2}\left(0, t ; L^{2}(Z)\right)}^{2} .
\end{aligned}
$$

For the second integral involving $v^{\star}$, integration by parts implies that

$$
\begin{aligned}
& \frac{1}{2} \int_{0}^{t} \int_{Z}\left(\underline{\underline{v}}^{\star} \cdot(B \nabla)\right) \sum_{i=1}^{2 n} \dot{\lambda}_{i} \phi_{i} \cdot \underline{u} \\
& \quad=-\frac{1}{2} \int_{0}^{t} \int_{Z} \sum_{j=1}^{3}\left(\partial_{j}\left(B^{\top} \underline{v}^{\star}\right)_{j} \underline{u}\right) \cdot \sum_{i=1}^{2 n} \dot{\lambda}_{i} \phi_{i}+\frac{1}{2} \int_{0}^{t} \int_{\partial Z}\left(\underline{u} \cdot \sum_{i=1}^{2 n} \dot{\lambda}_{i} \phi_{i}\right)\left(\underline{v}^{\star} \cdot B n_{Z}\right) .
\end{aligned}
$$

The first integral on the right-hand side can be estimated as

$$
\left|\frac{1}{2} \int_{0}^{t} \int_{Z} \sum_{j=1}^{3}\left(\partial_{j}\left(B^{\top} \underline{v}^{\star}\right) j \underline{u}\right) \cdot \sum_{i=1}^{2 n} \dot{\lambda}_{i} \phi_{i}\right| \leq C+\frac{1}{8}\left\|\sum_{i=1}^{2 n} \dot{\lambda}_{i} \phi_{i} \sqrt{1+\delta^{\star}}\right\|_{L^{2}\left(0, t ; L^{2}(Z)\right)}^{2},
$$

while, for the second one, we can write

$$
\begin{aligned}
\left|\frac{1}{2} \int_{0}^{t} \int_{\partial Z}\left(\underline{u} \cdot \sum_{i=1}^{2 n} \dot{\lambda}_{i} \phi_{i}\right)\left(\underline{v}^{\star} \cdot B n_{Z}\right)\right| & =\left|\frac{1}{2} \int_{0}^{t} \int_{\omega} \partial_{t} h \partial_{t}^{2} h\left(\underline{v}^{\star} \cdot B n_{Z}\right)\right| \\
& \leq C+\frac{1}{8}\left\|\partial_{t}^{2} h\right\|_{L^{2}\left(0, t ; L^{2}(\omega)\right)^{\prime}}^{2}
\end{aligned}
$$

where we used that $\underline{u}=\partial_{t} h, \partial_{t} \underline{u}=\sum_{i=1}^{2 n} \dot{\lambda}_{i} \phi_{i}=\partial_{t}^{2} h$ on $\partial Z \backslash \Gamma_{0}$ and that, by (39), $\partial_{t} h$ is bounded in $L^{2}\left(0, t ; L^{2}(\omega)\right)$.

For the next term in (41), we easily have

$$
\left|\int_{0}^{t} \int_{Z} \partial_{3} \underline{u} \cdot \sum_{i=1}^{2 n} \dot{\lambda}_{i} \phi_{i} z \partial_{t} \delta^{\star}\right| \leq C+\frac{1}{8}\left\|\sum_{i=1}^{2 n} \dot{\lambda}_{i} \phi_{i} \sqrt{1+\delta^{\star}}\right\|_{L^{2}\left(0, t ; L^{2}(Z)\right)}^{2} .
$$

Concerning integrals involving $h$, we start with the estimate

$$
\left|\frac{1}{2} \int_{0}^{t} \int_{\omega} \partial_{t} h \partial_{t} \delta^{\star} \partial_{t}^{2} h\right| \leq C+\frac{1}{8}\left\|\partial_{t}^{2} h\right\|_{L^{2}\left(0, t ; L^{2}(\omega)\right)}^{2} .
$$


The term $\int_{0}^{t}\left\|\Delta \partial_{t} h\right\|_{L^{2}(\omega)}^{2}$ is bounded in view of (39). For the next term, we have, due to $H^{1}\left(0, T ; L^{2}(\omega)\right) \hookrightarrow L^{\infty}\left(0, T ; L^{2}(\omega)\right)$ and $(39)$, that

$$
\left|\int_{\omega} \Delta h(t) \Delta \partial_{t} h(t)\right| \leq \frac{1}{4}\left\|\Delta \partial_{t} h(t)\right\|_{L^{2}(\omega)}^{2}+C\|\Delta h(t)\|_{L^{2}(\omega)}^{2} \leq \frac{1}{4}\left\|\Delta \partial_{t} h(t)\right\|_{L^{2}(\omega)}^{2}+C .
$$

Moreover, due to the boundedness of $\omega$,

$$
\left|\int_{0}^{t} \int_{\omega} \kappa G\left(\partial_{t} h\right) \partial_{t}^{2} h\right| \leq \int_{0}^{t} \int_{\omega} \kappa \cdot\left|\partial_{t}^{2} h\right| \leq C \kappa t+\frac{1}{8}\left\|\partial_{t}^{2} h\right\|_{L^{2}\left(0, t ; L^{2}(\omega)\right)}^{2} .
$$

Finally, the estimate

$$
\left|\int_{0}^{t} \int_{Z} \frac{f}{2 n} \sum_{i=1}^{2 n} \dot{\lambda}_{i} \phi_{i}\left(1+\delta^{\star}\right)\right| \leq C+\frac{1}{8}\left\|\sum_{i=1}^{2 n} \dot{\lambda}_{i} \phi_{i} \sqrt{1+\delta^{\star}}\right\|_{L^{2}\left(0, t ; L^{2}(\omega)\right)}^{2}
$$

holds. So, in total, we conclude

$$
\left\|\sum_{i=1}^{2 n} \dot{\lambda}_{i} \phi_{i} \sqrt{1+\delta^{\star}}\right\|_{L^{2}\left(0, t ; L^{2}(Z)\right)}^{2}+\left\|\partial_{t}^{2} h\right\|_{L^{2}\left(0, t ; L^{2}(\omega)\right)}^{2}+\left\|\Delta \partial_{t} h(t)\right\|_{L^{2}(\omega)}^{2} \leq C .
$$

Due to $\partial_{t} \underline{u}=\sum_{i=1}^{2 n} \dot{\lambda}_{i} \phi_{i}+\sum_{i=1}^{2 n} \lambda_{i} \partial_{t} \phi_{i}$, with the second term bounded in $L^{2}\left(0, T ; L^{2}(Z)\right)$ because of (43), (45) and (46), and $\sqrt{1+\delta^{\star}} \geq \sqrt{\frac{m}{2}}$, we have shown-in the original notation, that is to say, with indices $n$ and $\varepsilon$ - that

$$
\left\|\partial_{t} \underline{u_{\varepsilon}^{n}}\right\|_{L^{2}\left(0, T ; L^{2}(Z)\right)}+\left\|\partial_{t}^{2} h_{\varepsilon}^{n}\right\|_{L^{2}\left(0, T ; L^{2}(\omega)\right)} \leq \widetilde{C}(\varepsilon) .
$$

Step 5: Convergence of $u_{\varepsilon}^{n}, h^{n}$ to a weak solution

The bounds from (39) and (48) imply that there is a subsequence of $\left(\left(\underline{u_{\varepsilon}^{n}}, h_{\varepsilon}^{n}\right)_{n \in \mathbb{N}}\right)$, which we denote by $\left(\left(u_{\varepsilon}^{n}, h_{\varepsilon}^{n}\right)_{n \in \mathbb{N}}\right)$ again such that

$$
\begin{aligned}
\partial_{t} \underline{u_{\varepsilon}^{n}}-\partial_{t} \underline{u_{\varepsilon}} & \text { in } L^{2}\left(0, T ; L^{2}(Z)\right), \\
\underline{u_{\varepsilon}^{n}}-\underline{u_{\varepsilon}} & \text { in } L^{2}\left(0, T ; H^{1}(Z)\right), \\
\partial_{t}^{2} h_{\varepsilon}^{n} \rightarrow \partial_{t}^{2} h_{\varepsilon} & \text { in } L^{2}\left(0, T ; L^{2}(\omega)\right), \\
\Delta h_{\varepsilon}^{n} \rightarrow \Delta h_{\varepsilon} & \text { in } H^{1}\left(0, T ; L^{2}(\omega)\right), \\
\partial_{t} h_{\varepsilon}^{n} \rightarrow \partial_{t} h_{\varepsilon} & \text { in } L^{2}\left(0, T ; L^{2}(\omega)\right), \\
\partial_{t} h_{\varepsilon}^{n} \rightarrow \partial_{t} h_{\varepsilon} & \text { pointwise a.e. on }(0, T) \times \omega
\end{aligned}
$$

as $n \rightarrow \infty$ for some

$$
\left(u_{\varepsilon}, h_{\varepsilon}\right) \in L^{2}\left(0, T ; H_{0, \Gamma_{0}}^{1}(Z)\right) \times\left(H^{2}\left(0, T ; L^{2}(\omega)\right) \cap H^{1}\left(0, T ; H_{0}^{2}(\omega)\right)\right) .
$$

The last convergence in (49) is implied by the second to last (up to a subsequence), which, in turn, is implied by $(49)_{3,4}$ and the Aubin-Lions lemma. Using the above convergences, we let $n$ tend to infinity in (35) and (36) after an integration in time. For the convergence

$$
\int_{0}^{t} \int_{\omega} \kappa G_{\varepsilon}\left(\partial_{t} h_{\varepsilon}^{n}\right) \xi_{j} \longrightarrow \int_{0}^{t} \int_{\omega} \kappa G_{\varepsilon}\left(\partial_{t} h_{\varepsilon}\right) \xi_{j} \text { as } n \rightarrow \infty
$$

for all $j \in \mathbb{N}$, we use that $\left|G_{\varepsilon}\left(\partial_{t} h_{\varepsilon}^{n}\right)\right| \leq 1$, the convergence of $\left(\partial_{t} h_{\varepsilon}^{n}\right)_{n \in \mathbb{N}}$ to $\partial_{t} h_{\varepsilon}$ pointwise almost everywhere on $(0, T) \times \omega$ and the dominated convergence theorem. 
For the interface condition, we note that by construction, as in (37), we have

$$
\underline{u_{\varepsilon}^{n}}(t, x, y, 1)=\left(0,0, \partial_{t} h_{\varepsilon}^{n}(t, x, y)\right)^{\top} \text { a.e. on }(0, T) \times \omega \forall n \in \mathbb{N} .
$$

The first two convergences in (49) imply via the Aubin-Lions lemma that, as $n \rightarrow \infty$, we have $\underline{u_{\varepsilon}^{n}} \rightarrow \underline{u_{\varepsilon}}$ in $L^{2}\left(0, T ; H^{3 / 4}(Z)\right)$; thus,

$$
{\underline{u_{\varepsilon}^{n}}}_{\mid \omega \times\{1\}} \rightarrow \underline{u_{\varepsilon} \mid \omega \times\{1\}} \text { in } L^{2}\left(0, T ; L^{2}(\omega)\right) .
$$

We already know that $\partial_{t} h_{\varepsilon}^{n} \rightarrow \partial_{t} h_{\varepsilon}$ in $L^{2}\left(0, T ; L^{2}(\omega)\right)$ as $n \rightarrow \infty$. Therefore, by possibly choosing a suitable subsequence, we deduce

$$
\underline{u_{\varepsilon}}(t, x, y, 1)=\left(0,0, \partial_{t} h_{\mathcal{E}}(t, x, y)\right)^{\top} \text { a.e. on }(0, T) \times \omega .
$$

For the initial values, we have by design for $n \rightarrow \infty$

$$
\begin{aligned}
\underline{u_{\varepsilon}^{n}}(0) & =\underline{u_{\varepsilon, 0}^{n}} \longrightarrow \underline{u_{0}^{\varepsilon}} \text { in } L^{2}(Z) \\
\partial_{t} h_{\varepsilon}^{n}(0) & =\overline{h_{\varepsilon, 1}^{n}} \longrightarrow h_{\varepsilon}^{1} \text { in } L^{2}(\omega) .
\end{aligned}
$$

Due to $H^{1}\left(0, T ; L^{2}(X)\right) \hookrightarrow C\left([0, T] ; L^{2}(X)\right)$ with $X \in\{\omega, Z\}$, we deduce that $\underline{u_{\varepsilon}}(0)=\underline{u_{0}^{\varepsilon}}$ and $h_{\varepsilon}(0)=h_{0}^{\varepsilon}$.

Now, in order to combine the equations (35) and (36), and to solve the weak formulation (32) on $Z$ as a whole, we take a relevant test function $\left(\underline{\phi_{\varepsilon}}, b\right) \in L^{2}\left(0, T ; H_{0, \Gamma_{0}}^{1}(Z)\right) \times$ $L^{2}\left(0, T ; H_{0}^{2}(\omega)\right)$, as in (32).

Let $P_{N}(b)=\sum_{j=1}^{N} \tilde{b}_{j}(t) \xi_{j}$ denote the projection of $b$ onto $\operatorname{span}\left(\xi_{j}\right)_{j \in\{1, \ldots, N\}}$. As before, $\phi_{j}^{1, \varepsilon}$ denotes the solution of the stationary Stokes-like system (33), and $\phi_{b}^{1, \varepsilon}$ denotes the weak solution to the similar system but with boundary value $(0,0, b)^{\top}$ instead of $\left(0,0, \xi_{j}\right)^{\top}$. Due to the linearity of this system and the continuous dependence of solutions on the data, we get

$$
\left\|\sum_{j=1}^{N} \tilde{b}_{j} \phi_{j}^{1, \varepsilon}-\phi_{b}^{1, \varepsilon}\right\|_{L^{2}\left(0, T ; H^{1}(Z)\right)} \leq C\left\|P_{N}(b)-b\right\|_{L^{2}\left(0, T ; H^{1 / 2}(\omega)\right)} \rightarrow 0 \text { as } N \rightarrow \infty .
$$

This enables us to multiply (36) with $\tilde{b}_{j}(t)$, integrate in time, take the limit $n$ to infinity for the solution, as justified above, take the sum over $j \in\{1, \ldots, N\}$, and then consider the limit $N \rightarrow \infty$ for the test function. This allows us to basically replace $\xi_{j}$ with $b$ and $\phi_{j}^{1, \varepsilon}$ with $\phi_{b}^{1, \varepsilon}$ in (36).

Then, we consider the Dirichlet part $\phi^{0}:=\underline{\phi_{\varepsilon}}-\phi_{b}^{1, \varepsilon}$ which satisfies $\phi^{0}=0$ on $\partial Z$ and $\operatorname{div}\left(B_{\varepsilon}^{\top}\left(\phi^{0}\right)\right)=0$. For this reason, we can consider the projection of $P_{M}\left(\phi^{0}\right)=$ $\sum_{i=1}^{M} \tilde{\alpha}_{i}(t) \phi_{i}^{0, \varepsilon}$ onto $\operatorname{span}\left(\phi_{i}^{0, \varepsilon}\right)_{i \in\{1, \ldots, M\}}$. Analogously to before, we multiply (35) with $\tilde{\alpha}_{i}(t)$, integrate over time, take the limit $n \rightarrow \infty$ for the solution, take the sum over $i \in\{1, \ldots, M\}$, and then take the limit $M \rightarrow \infty$ for the test function.

Next using that $\phi_{\varepsilon}=\phi^{0}+\phi_{b}^{1, \varepsilon}$, we can add the two resulting equations to get that $\left(\underline{u_{\varepsilon}}, h_{\varepsilon}\right)$ solves (28). Since the diffeomorphism $\chi_{\varepsilon}$ is smooth, we obtain a weak solution for the problem (28), formulated on $\Omega_{\delta_{\varepsilon}^{\star}}$, by setting $\left(u_{\varepsilon}, h_{\varepsilon}\right)=\left(\underline{u}_{\varepsilon} \circ \chi_{\varepsilon}^{-1}, h_{\varepsilon}\right)$.

Furthermore, weak solutions of (28) are unique. For the proof, take two solutions $\left(u_{\varepsilon}^{1}, h_{\varepsilon}^{1}\right),\left(u_{\varepsilon}^{2}, h_{\varepsilon}^{2}\right)$ of $(28)$ to the same given data and initial values. In the equation that 
is solved by the difference $\left(u_{\varepsilon}^{1}-u_{\varepsilon}^{2}, h_{\varepsilon}^{1}-h_{\varepsilon}^{2}\right)$, we test with the difference itself. After integration by parts, we obtain

$$
\begin{aligned}
& \frac{1}{2} \int_{\Omega_{\delta_{\varepsilon}^{\star}(t)}}\left|\left(u_{\varepsilon}^{1}-u_{\varepsilon}^{2}\right)(t)\right|^{2}+\mu \int_{0}^{t} \int_{\Omega_{\delta_{\varepsilon}^{\star}(s)}}\left|\nabla\left(u_{\varepsilon}^{1}-u_{\varepsilon}^{2}\right)\right|^{2} \\
& \quad+\frac{1}{2} \int_{\omega}\left|\partial_{t} \Delta\left(h_{\varepsilon}^{1}-h_{\varepsilon}^{2}\right)(t)\right|^{2}+\frac{1}{2} \int_{\omega}\left|\Delta\left(h_{\varepsilon}^{1}-h_{\varepsilon}^{2}\right)(t)\right|^{2}+\int_{0}^{t} \int_{\omega}\left|\Delta \partial_{t}\left(h_{\varepsilon}^{1}-h_{\varepsilon}^{2}\right)\right|^{2} \\
& \quad+\int_{0}^{t} \int_{\omega} \kappa\left(G_{\varepsilon}\left(\partial_{t} h_{\varepsilon}^{1}\right)-G_{\varepsilon}\left(\partial_{t} h_{\varepsilon}^{2}\right)\right)\left(\partial_{t} h_{\varepsilon}^{1}-\partial_{t} h_{\varepsilon}^{2}\right)=0 .
\end{aligned}
$$

Since $G_{\varepsilon}$ is the derivative of a convex function, it is monotonically increasing. Therefore, every term on the left-hand side is non-negative, and we conclude that $u_{\varepsilon}^{1}=u_{\varepsilon}^{2}$ and $\partial_{t} h_{\varepsilon}^{1}=\partial_{t} h_{\varepsilon}^{2}$. The latter implies $h_{\varepsilon}^{1}=h_{\varepsilon}^{2}$ due to $h_{\varepsilon}^{1}(0)=h_{\varepsilon}^{2}(0)$.

\section{Existence of Weak Solutions: The Fixed Point Argument}

Step 6: The fixed point problem of $F_{\varepsilon}$

Given initial values $u_{0}^{\varepsilon}, h_{0}^{\varepsilon}$, and $h_{1}^{\varepsilon}$, for any pair $(\delta, v)$ with $\delta \in H^{1}\left(0, T ; C^{0}(\bar{\omega}) \cap H_{0}^{1}(\omega)\right)$, $M \geq 1+\delta \geq m>0$ on $[0, T] \times \bar{\omega}$ and $v \in L^{2}\left(0, T ; L^{2}\left(B_{2 M}\right)\right)$, we have shown in Section 5 the existence of a weak unique solution $\left(u_{\varepsilon}, h_{\varepsilon}\right)$ to the "linearized" problem (28) with $v_{\varepsilon}^{\star}=R_{\epsilon}(v)$ and $\delta_{\varepsilon}^{\star}=N_{\epsilon}(\delta)$ where $\delta_{\mid t=0}=h_{0}^{\varepsilon}$. This solution satisfies the estimates, cf. the $a$ priori estimates (31) and (40)

$$
\begin{aligned}
& \left\|u_{\mathcal{\varepsilon}}\right\|_{L^{\infty}\left(0, T ; L^{2}\left(\Omega_{\delta_{\varepsilon}^{\star}(t)}\right)\right.}+\left\|\nabla u_{\mathcal{E}}\right\|_{L^{2}\left(0, T ; L^{2}\left(\Omega_{\delta_{\varepsilon}^{\star}(t)}\right)\right.}+\left\|\partial_{t} h_{\mathcal{E}}\right\|_{L^{\infty}\left(0, T ; L^{2}(\omega)\right)}+\left\|\Delta h_{\mathcal{\varepsilon}}\right\|_{H^{1}\left(0, T ; L^{2}(\omega)\right)} \\
& \quad \leq C\left(T,\left\|u_{0}\right\|_{L^{2}\left(\Omega_{h_{0}}\right)},\|f\|_{L^{2}\left((0, T) \times \mathbb{R}^{3}\right)}\left\|h_{0}\right\|_{H_{0}^{2}(\omega)}\left\|h_{1}\right\|_{L^{2}(\omega)}\right)
\end{aligned}
$$

with a constant $C>0$ independent of $\varepsilon>0$ and

$$
\left\|\partial_{t} \underline{u_{\varepsilon}}\right\|_{L^{2}\left(0, T ; L^{2}(Z)\right)}+\left\|\partial_{t}^{2} h_{\varepsilon}\right\|_{L^{2}\left(0, T ; L^{2}(\omega)\right)} \leq \widetilde{C}(\varepsilon),
$$

as in (48), the corresponding weak convergences in (49) and the weak lower semi-continuity of the norm. For $T^{*} \in(0, T]$, let

$$
Y:=H^{1}\left(0, T^{*} ; C^{0}(\bar{\omega}) \cap H_{0}^{1}(\omega)\right) \times L^{2}\left(0, T^{*} ; L^{2}\left(B_{2 M}\right)\right) .
$$

Then, we consider the solution operator

$$
F_{\varepsilon}: \mathcal{B}_{M}^{m, \varepsilon} \rightarrow Y, \quad(\delta, v) \mapsto\left(h_{\varepsilon}, \bar{u}_{\varepsilon}\right),
$$

where

$$
\begin{aligned}
\mathcal{B}_{M}^{m, \varepsilon}:=\{ & (\delta, v) \in Y \mid\|(\delta, v)\|_{Y} \leq C_{M}, \\
& \left.m \leq 1+\delta(t, x, y) \leq M \text { on }\left[0, T^{*}\right] \times \bar{\omega}, \delta_{\mid t=0}=h_{0}^{\varepsilon}\right\},
\end{aligned}
$$

and $\left(u_{\varepsilon}, h_{\varepsilon}\right)$ denotes the solution constructed in the previous steps for the data $(\delta, v)$ and with

$$
\bar{u}_{\varepsilon}= \begin{cases}u_{\varepsilon} & \text { in } \Omega_{\delta_{\varepsilon}^{\star}(t)}, \\ \left(0,0, \partial_{t} h_{\varepsilon}\right)^{\top} & \text { in } B_{2 M} \backslash \Omega_{\delta_{\varepsilon}^{\star}(t)} .\end{cases}
$$

It is easy to check that $\mathcal{B}_{M}^{m, \varepsilon}$ is non-empty, closed and convex. If we can show that $F_{\varepsilon}\left(\mathcal{B}_{M}^{m, \varepsilon}\right) \subset$ $\mathcal{B}_{M}^{m, \varepsilon}$, that $F_{\varepsilon}\left(\mathcal{B}_{M}^{m, \varepsilon}\right)$ is relatively compact and that $F_{\varepsilon}$ is continuous, we get the existence of at least one fixed point by Schauder's fixed-point theorem, as in Reference [20] (Corollary 10.2). Note that $m>0$ was already chosen such that $\min _{\bar{w}}\left(1+h_{0}\right) \geq 2 m>0$, but $M$ and $T^{*}$ still have to be chosen large and small enough, respectively, such that $F_{\varepsilon}$ satisfies the mentioned properties, if $C_{M}$ is chosen large enough, as well. 


\section{Step 6.1: $F_{\varepsilon}$ is a self-map}

First, we want to show that $F_{\varepsilon}\left(\mathcal{B}_{M}^{m, \varepsilon}\right) \subset \mathcal{B}_{M}^{m, \varepsilon}$ if $M>0$ is large enough. Due to (50) and $H^{1}\left(0, T ; H^{2}(\omega)\right) \hookrightarrow C^{0}([0, T] \times \bar{\omega})$, we conclude that, if $M$ is chosen large enough according to the given data, then

$$
\sup _{[0, T] \times \bar{\omega}}\left(1+h_{\varepsilon}\right) \leq M
$$

Furthermore, it is easy to see that

$$
\left\|\bar{u}_{\varepsilon}\right\|_{L^{2}\left(0, T ; L^{2}\left(B_{2 M}\right)\right)} \leq\left\|u_{\varepsilon}\right\|_{L^{2}\left(0, T ; L^{2}\left(\Omega_{\delta_{\varepsilon}^{\star}(t)}\right)\right)}+2 M\left\|\partial_{t} h_{\varepsilon}\right\|_{L^{2}\left(0, T ; L^{2}(\omega)\right)} \stackrel{(50)}{\leq} C_{1} \cdot M .
$$

Due to $h_{\varepsilon} \in H^{1}\left(0, T ; H^{2}(\omega)\right)$ and the embedding $H^{2}(\omega) \hookrightarrow C^{0}(\bar{\omega})$, we also get from (50) that $\left\|h_{\varepsilon}\right\|_{H^{1}\left(0, T ; C^{0}(\bar{\omega}) \cap H_{0}^{1}(\omega)\right)} \leq C_{2}$. Hence,

$$
\left\|\left(h_{\varepsilon}, \bar{u}_{\varepsilon}\right)\right\|_{Y} \leq C_{1} \cdot M+C_{2}=: C_{M} .
$$

Since $h_{\varepsilon}$ is bounded in $H^{1}\left(0, T ; H^{2}(\omega)\right), H^{1}\left(0, T ; H^{2}(\omega)\right) \hookrightarrow C([0, T] \times \bar{\omega})$ and $\min _{\bar{\omega}}\left(1+h_{0}^{\varepsilon}\right) \geq \frac{3 m}{2}$, as in (22), there is a time $T^{*}>0$ such that

$$
m \leq \min _{\left[0, T^{*}\right] \times \bar{\omega}}\left(1+h_{\varepsilon}\right)
$$

for $\varepsilon>0$ small enough. In total, we have verified that $F_{\varepsilon}\left(\mathcal{B}_{M}^{m, \varepsilon}\right) \subset \mathcal{B}_{M}^{m, \varepsilon}$.

Step 6.2: $F_{\varepsilon}\left(\mathcal{B}_{M}^{m, \varepsilon}\right)$ is relatively compact

Let $\left(\delta_{n}, v_{n}\right)_{n \in \mathbb{N}} \subset \mathcal{B}_{M}^{m, \varepsilon}$, and let $\left(h_{\varepsilon}^{n}, u_{\varepsilon}^{n}\right)=F_{\varepsilon}\left(\delta_{n}, v_{n}\right)$ denote the solution to (28). Due to (50) and (51), $\left(\partial_{t} h_{\varepsilon}^{n}\right)_{n}$ is bounded in $L^{2}\left(0, T^{*} ; H_{0}^{2}(\omega)\right)$, and $\left(\partial_{t}^{2} h_{\varepsilon}^{n}\right)_{n}$ is bounded in $L^{2}\left(0, T^{*} ; L^{2}(\omega)\right)$. Since $H_{0}^{2}(\omega)$ is compactly embedded in $H_{0}^{1}(\omega) \cap C^{0}(\bar{\omega})$, we get that $\left(\partial_{t} h_{\varepsilon}^{n}\right)_{n}$ is relatively compact in $L^{2}\left(0, T^{*} ; H^{1}(\omega) \cap C^{0}(\bar{\omega})\right)$. Moreover, we have that $\left(\underline{u}_{\varepsilon}{ }^{n}\right)_{n}$ is bounded in $L^{2}\left(0, T^{*} ; H^{1}(Z)\right)$ and $\left(\partial_{t} \underline{u_{\varepsilon}}{ }^{n}\right)_{n}$ is bounded in $L^{2}\left(0, T^{*} ; L^{2}(Z)\right)$. Hence, $\left(\underline{u_{\varepsilon}}{ }^{n}\right)_{n}$ is relatively compact in $L^{2}\left(0, T^{*} ; L^{2}(Z)\right)$. Let $\underline{u_{\varepsilon}}$ denote the limit of the corresponding subsequence.

Since $\left(\delta_{n}, v_{n}\right) \in \mathcal{B}_{M}^{m, \varepsilon}$ we have for the corresponding regularization $\delta_{n, \varepsilon}^{\star}=N_{\varepsilon}\left(\delta_{n}\right)$ of $\delta_{n}$ (see (24) and (25) for the definition and some properties)

$$
\begin{aligned}
\left\|\delta_{n, \varepsilon}^{\star}\right\|_{H^{1}\left(0, T^{*} ; H^{1}(\omega)\right)} & \leq\left\|S_{\varepsilon}\left(\delta_{n}-h_{0}^{\varepsilon}\right)-\left(\delta_{n}-h_{0}^{\varepsilon}\right)\right\|_{H^{1}\left(0, T^{*} ; H^{1}(\omega)\right)}+\left\|\delta_{n}\right\|_{H^{1}\left(0, T^{*} ; H^{1}(\omega)\right)} \\
& \leq 2 C_{M}
\end{aligned}
$$

for all $n \in \mathbb{N}$ and $\varepsilon>0$ small enough. Since the embedding $H^{1}\left(0, T^{*} ; H^{1}(\omega)\right) \hookrightarrow$ $L^{2}\left(\left(0, T^{*}\right) \times \omega\right)$ is compact, there exists a subsequence of $\left(\delta_{n, \varepsilon}^{\star}\right)_{n \in \mathbb{N}}$, called again $\left(\delta_{n, \varepsilon}^{\star}\right)_{n \in \mathbb{N}}$, that converges pointwise a.e. on $\left(0, T^{*}\right) \times \omega$ to an element $\tilde{\delta}_{\varepsilon} \in H^{1}\left(0, T^{*} ; H^{1}(\omega)\right)$ as $n \rightarrow \infty$. Due to

$$
2 M \geq 1+\delta_{n, \varepsilon}^{\star} \geq \frac{m}{2} \text { on }[0, T] \times \bar{\omega},
$$

as in (26), the same estimate holds for the limit $\tilde{\delta}_{\varepsilon}$. Now, let

$$
\chi_{\varepsilon}^{n}(t): Z \longrightarrow \Omega_{\delta_{n, \varepsilon}^{\star}(t)},(x, y, z) \mapsto\left(x, y, z\left(1+\delta_{n, \varepsilon}^{\star}(t, x, y)\right)\right)^{\top} \forall t \in\left(0, T^{*}\right)
$$

and define $\chi_{\varepsilon}(t)$ similarly but with $\tilde{\delta}_{\varepsilon}$ instead of $\delta_{n, \varepsilon}^{\star}$. We want to show that

$$
\chi_{\Omega_{\delta_{n, \varepsilon}^{\star}(t)}}\left(\underline{u_{\varepsilon}} \circ\left(\chi_{\varepsilon}^{n}\right)^{-1}\right) \rightarrow \chi_{\Omega_{\tilde{\delta}_{\varepsilon}(t)}}\left(\underline{u_{\varepsilon}} \circ \chi_{\varepsilon}^{-1}\right) \text { in } L^{2}\left(0, T^{*} ; L^{2}\left(B_{2 M}\right)\right) \text { as } n \rightarrow \infty \text {. }
$$


Concerning the convergence of the norms, we have that

$$
\begin{aligned}
& \int_{(0, T) \times B_{2 M}}\left|\chi_{\Omega_{\delta_{n, \varepsilon}^{\star}(t)}}\left(\underline{u_{\varepsilon}} \circ\left(\chi_{\varepsilon}^{n}\right)^{-1}\right)\right|^{2}=\int_{(0, T) \times \Omega_{\delta_{n, \varepsilon}^{\star}(t)}}\left|\underline{u_{\varepsilon}} \circ\left(\chi_{\varepsilon}^{n}\right)^{-1}\right|^{2} \\
= & \int_{(0, T) \times \Omega_{\tilde{\delta}_{\varepsilon}(t)}}\left|\underline{u_{\varepsilon}} \circ\left(\chi_{\varepsilon}\right)^{-1}\right|^{2} \operatorname{det}\left(\nabla\left(\chi_{\varepsilon}^{n} \circ \chi_{\varepsilon}^{-1}\right)\right)=\int_{(0, T) \times \Omega_{\tilde{\delta}_{\varepsilon}(t)}}\left|\underline{u_{\varepsilon}} \circ\left(\chi_{\varepsilon}\right)^{-1}\right|^{2} \frac{1+\delta_{n, \varepsilon}^{\star}}{1+\tilde{\delta}_{\varepsilon}} .
\end{aligned}
$$

Due to the pointwise convergence of $\left(\delta_{n, \varepsilon}^{\star}\right)_{n \in \mathbb{N}}$ to $\tilde{\delta}_{\varepsilon}$ a.e. on $(0, T) \times \omega$ as $n \rightarrow \infty$

$$
\frac{1+\delta_{n, \varepsilon}^{\star}}{1+\tilde{\delta}_{\varepsilon}} \leq \frac{2 M}{m / 2}
$$

the dominated convergence theorem yields the convergence of the norms. The weak convergence follows in a similar fashion. In total, we obtain the strong convergence (53). Hence, (53), in combination with

$$
\begin{gathered}
\left\|\chi_{\Omega_{\delta_{n, \varepsilon}^{\star}(t)}}\left(u_{\varepsilon}^{n}-\underline{u_{\varepsilon}} \circ\left(\chi_{\varepsilon}^{n}\right)^{-1}\right)\right\|_{L^{2}\left(\left(0, T^{*}\right) \times B_{2 M}\right)}=\left\|u_{\varepsilon}^{n}-\underline{u_{\varepsilon}} \circ\left(\chi_{\varepsilon}^{n}\right)^{-1}\right\|_{L^{2}\left(0, T^{*} ; L^{2}\left(\Omega_{\delta_{n, \varepsilon}^{\star}(t)}\right)\right)} \\
=\left\|\left(\underline{u_{\varepsilon}}{ }^{n}-\underline{u_{\varepsilon}}\right) \sqrt{1+\delta_{n, \varepsilon}^{\star}}\right\|_{L^{2}\left(0, T^{*} ; L^{2}(Z)\right)} \leq \sqrt{2 M}\left\|\underline{u_{\varepsilon}}-\underline{u_{\varepsilon}}\right\|_{L^{2}\left(0, T^{*} ; L^{2}(Z)\right)} \longrightarrow 0
\end{gathered}
$$

as $n \rightarrow \infty$, implies that $\left(\chi_{\Omega_{\delta_{n, \varepsilon}^{\star}(t)}} u_{\varepsilon}^{n}\right)_{n \in \mathbb{N}}$ converges in $L^{2}\left(0, T^{*} ; L^{2}\left(B_{2 M}\right)\right)$. Therefore, since $\left(\chi_{\Omega_{\delta_{n, \varepsilon}^{\star}(t)}} u_{\varepsilon}^{n}\right)$ is relatively compact in $L^{2}\left(0, T^{*} ; L^{2}\left(B_{2 M}\right)\right)$ and $\left(\partial_{t} h_{\varepsilon}^{n}\right)_{n \in \mathbb{N}}$ is relatively compact in $L^{2}\left(0, T^{*} ; H_{0}^{1}(\omega) \cap C^{0}(\omega)\right)$, as previously shown, we also deduce that $\left(\bar{u}_{\varepsilon}^{n}\right)_{n \in \mathbb{N}}$ is relatively compact in $L^{2}\left(\left(0, T^{*}\right) \times B_{2 M}\right)$. This implies that $F_{\varepsilon}\left(\mathcal{B}_{M}^{m, \varepsilon}\right) \subset Y$ is relatively compact.

Step 6.3: $F_{\varepsilon}$ is continuous

Eventually, we prove that $F_{\varepsilon}$ is continuous. Let $\left(\delta_{n}, v_{n}\right)_{n} \subset \mathcal{B}_{M}^{m, \varepsilon}$ be a sequence converging to $(\delta, v)$ in $Y$ as $n \rightarrow \infty$. We want to show that any subsequence of $\left(F_{\varepsilon}\left(\delta_{n}, v_{n}\right)\right)_{n \in \mathbb{N}}$ has a subsequence that converges to $F_{\varepsilon}(\delta, v)$, which implies that $\left(F_{\varepsilon}\left(\delta_{n}, v_{n}\right)\right)_{n \in \mathbb{N}}$ converges to $F_{\varepsilon}(\delta, v)$. Since any subsequence of $\left(\delta_{n}, v_{n}\right)_{n \in \mathbb{N}}$ converges to the same limit $(\delta, v)$ in $Y$, too, it is enough to consider only the (sub)sequence $\left(F_{\mathcal{\varepsilon}}\left(\delta_{n}, v_{n}\right)\right)_{n \in \mathbb{N}}$.

We have already shown that a subsequence of $F_{\varepsilon}\left(\delta_{n}, v_{n}\right)=:\left(h_{\varepsilon}^{n}, \bar{u}_{\varepsilon}^{n}\right)$, which we assume to coincide with the sequence itself again, converges to an element $\left(\tilde{h}_{\varepsilon}, \tilde{\bar{u}}_{\varepsilon}\right)$ in $Y$. Moreover, since $\left(h_{\varepsilon}^{n}, \bar{u}_{\varepsilon}^{n}\right)$ is a solution of the "linearized" system (28), it is also bounded due to the $a$ priori estimates (50) and (51). This also implies that (up to a subsequence) $\left(h_{\varepsilon}^{n}, \bar{u}_{\varepsilon}^{n}\right)_{n \in \mathbb{N}}$ converges weakly in $H^{1}\left(0, T^{*} ; H_{0}^{2}(\omega)\right) \times L^{2}\left(0, T^{*} ; H^{1}\left(B_{2 M}\right)\right)$ and $\left(\partial_{t}^{2} h_{\varepsilon}^{n}, \chi_{\Omega_{\delta_{n, \varepsilon}^{*}(t)}} \partial_{t} u_{\varepsilon}^{n}\right)$ converges weakly in $L^{2}\left(0, T^{*} ; L^{2}(\omega)\right) \times L^{2}\left(0, T ; L^{2}\left(B_{2 M}\right)\right)$.

So, we want to show that $\left(\tilde{h}_{\varepsilon}, \tilde{\bar{u}}_{\varepsilon}\right)=F_{\varepsilon}(\delta, v)$, i.e., $\left(\tilde{h}_{\varepsilon}, \tilde{\bar{u}}_{\varepsilon}\right)$ is a solution to the "linearized" problem (28) associated with $(\delta, v)$. Due to uniqueness, this indeed yields $\left(\tilde{h}_{\varepsilon}, \tilde{\tilde{u}}_{\varepsilon}\right)=F_{\varepsilon}(\delta, v)$. It is easy to see that the mentioned convergences imply

$$
\begin{aligned}
\tilde{\bar{u}}_{\varepsilon} & =\left(0,0, \partial_{t} \tilde{h}_{\varepsilon}\right)^{\top} & & \text { in } B_{2 M} \backslash \Omega_{\delta_{\varepsilon}^{\star}(t)}, \\
\tilde{\bar{u}}_{\mathcal{\varepsilon}}\left(t, x, y, 1+\delta_{\varepsilon}^{\star}(t, x, y)\right) & =\left(0,0, \partial_{t} \tilde{h}_{\varepsilon}(t, x, y)\right)^{\top} & & \text { on }\left[0, T^{*}\right] \times \omega, \\
\operatorname{div} \tilde{\bar{u}}_{\varepsilon} & =0 & & \text { in } B_{2 M} .
\end{aligned}
$$


Moreover, the system solved by $\left(h_{\varepsilon}^{n}, \bar{u}_{\varepsilon}^{n}\right)$ reads as follows: For all $\phi_{\varepsilon}^{n} \in V_{\delta_{\varepsilon}^{\star}}$, $b \in L^{2}\left(0, T^{*} ; H_{0}^{2}(\omega)\right)$ such that $\phi_{\varepsilon}\left(t, x, y, 1+\delta_{n, \varepsilon}^{\star}(t, x, y)\right)=(0,0, b(t, x, y))^{\top}$ on $\left[0, T^{*}\right] \times \omega$ we have, cf. (28),

$$
\begin{gathered}
\int_{0}^{t} \int_{\Omega_{\delta_{,, \varepsilon}^{\star}(s)}} \partial_{t} u_{\varepsilon}^{n} \cdot \phi_{\varepsilon}^{n}+\mu \int_{0}^{t} \int_{\Omega_{\delta_{n, \varepsilon}^{\star}(s)}} \nabla u_{\varepsilon}^{n}: \nabla \phi_{\varepsilon}^{n}+\frac{1}{2} \int_{0}^{t} \int_{\Omega_{\delta_{n, \varepsilon}^{\star},(s)}}\left(v_{n, \varepsilon}^{\star} \cdot \nabla\right) u_{\varepsilon}^{n} \cdot \phi_{\varepsilon}^{n} \\
\quad-\frac{1}{2} \int_{0}^{t} \int_{\Omega_{\delta_{n, \varepsilon}^{\star}(s)}}\left(v_{n, \varepsilon}^{\star} \cdot \nabla\right) \phi_{\varepsilon}^{n} \cdot u_{\varepsilon}^{n}+\frac{1}{2} \int_{0}^{t} \int_{\omega} \partial_{t} h_{\varepsilon}^{n}\left(\partial_{t} \delta_{n, \varepsilon}^{\star}\right) b+\int_{0}^{t} \int_{\omega} \partial_{t}^{2} h_{\varepsilon}^{n} b \\
\quad+\int_{0}^{t} \int_{\omega} \Delta \partial_{t} h_{\varepsilon}^{n} \Delta b+\int_{0}^{t} \int_{\omega} \Delta h_{\varepsilon}^{n} \Delta b+\int_{0}^{t} \int_{\omega} \kappa G_{\varepsilon}\left(\partial_{t} h_{\varepsilon}^{n}\right) b=\int_{0}^{t} \int_{\Omega_{\delta_{n, \varepsilon}^{\star}(s)} f \cdot \phi_{\varepsilon}^{n} .}
\end{gathered}
$$

We want to consider the limit as $n$ goes to infinity, but the test functions depend on $n$.

In order to overcome this problem, we first consider the test function

$$
\phi_{\varepsilon}^{0} \in C_{c}^{\infty}\left(\bigcup_{t \in\left(0, T^{*}\right)}\{t\} \times \Omega_{\delta_{\varepsilon}^{\star}(t)}\right), \operatorname{div} \phi_{\varepsilon}^{0}=0 .
$$

Then, $\left(\phi_{\varepsilon}^{0}, 0\right)$ is an admissible test function for large $n$ due to the convergence of $\left(\delta_{n}\right)_{n \in \mathbb{N}}$ to $\delta$ in $H^{1}\left(0, T^{*} ; C^{0}(\bar{\omega}) \cap H_{0}^{1}(\omega)\right)$, which implies uniform convergence; therefore, with the help of (24) for $n$ large enough,

$$
\phi_{\varepsilon}^{0}\left(t, x, y, 1+\delta_{n, \varepsilon}^{\star}(x, y)\right)=0 \text { on } \omega .
$$

For any trace funcion $b \in L^{2}\left(0, T^{*} ; H_{0}^{2}(\omega)\right)$ with $\int_{\omega} b=0$, there exists an element $z \in$ $L^{2}\left(0, T^{*} ; H^{1}\left(Z_{m / 2}\right)\right)\left(\right.$ where $\left.Z_{m / 2}=\omega \times\left(0, \frac{m}{2}\right)\right)$ such that

$$
\begin{array}{cl}
\operatorname{div} z & =0 \\
z_{\mid \partial Z_{m / 2}} & = \begin{cases}(0,0, b)^{\top} & \text { in }\left(0, T^{*}\right) \times Z_{m / 2}, \\
0 & \text { on }\left(0, T^{*}\right) \times \omega \times\left\{\frac{m}{2}\right\}\end{cases}
\end{array}
$$

and

$$
\|z\|_{L^{2}\left(0, T^{*} ; H^{1}\left(Z_{m / 2}\right)\right)} \leq c\|b\|_{L^{2}\left(0, T^{*} ; H^{1 / 2}(\omega)\right)} .
$$

Then, we consider

$$
\phi_{\varepsilon}^{1}(b):= \begin{cases}(0,0, b)^{\top} & \text { on } B_{2 M} \backslash Z_{m / 2} \\ z & \text { on } Z_{m / 2}\end{cases}
$$

The pair $\left(\phi_{\varepsilon}^{1}(b), b\right)$ is an admissible test function, too, since $1+\delta_{n, \varepsilon}^{\star} \geq \frac{m}{2}$ on $\left(0, T^{*}\right) \times \omega$ for all $n \in \mathbb{N}$.

For these special choices of test functions $\left(\phi_{\varepsilon}^{0}, 0\right)$ and $\left(\phi_{\varepsilon}^{1}(b), b\right)$ that do not depend on $n$, we can send $n$ to infinity in the equation. Here, we use the convergences of $\left(h_{\varepsilon}^{n}, u_{\varepsilon}^{n}\right)$, as we have done in the finite dimensional case. Let us remark that, in order to go to the limit in the most problematic term $\int_{0}^{t} \int_{\Omega_{\delta_{n, \varepsilon}^{\star}(s)}}\left(v_{n, \varepsilon}^{\star} \cdot \nabla\right) u_{\varepsilon}^{n} \cdot \phi_{\varepsilon}$, we deduce from (27) that

$$
\left\|v_{\varepsilon}^{\star}-v_{n, \varepsilon}^{\star}\right\|_{L^{\infty}\left((0, t) \times B_{2 M}\right)}=\left\|\varphi_{\varepsilon} * E_{0}\left(v-v_{n}\right)\right\|_{L^{\infty}\left((0, t) \times B_{2 M}\right)} \leq C_{\varepsilon}\left\|v-v_{n}\right\|_{L^{2}\left(0, T ; B_{2 M}\right)} \rightarrow 0
$$

as $n \rightarrow \infty$. So, we obtain that $\left(\tilde{h}_{\varepsilon}, \tilde{\bar{u}}_{\varepsilon}\right)$ is a weak solution to the limit problem-at least for the chosen set of test functions.

For a general test function $(\phi, b) \in V_{\delta_{\varepsilon}^{\star}} \times L^{2}\left(0, T^{*} ; H_{0}^{2}(\omega)\right)$ with

$$
\phi_{\varepsilon}\left(t, x, y, 1+\delta_{\varepsilon}^{\star}(t, x, y)\right)=(0,0, b(t, x, y))^{\top}
$$

on $\left[0, T^{*}\right] \times \omega$, we use that $\left(\phi_{\varepsilon}, b\right)=\left(\left(\phi_{\varepsilon}-\phi_{\varepsilon}^{1}(b), 0\right)+\left(\phi_{\varepsilon}^{1}(b), b\right)\right.$ such that the first summand $\phi_{\varepsilon}-\phi_{\varepsilon}^{1}(b)$ can be approximated by functions of the same type as $\phi_{\varepsilon}^{0}$. This shows that 
$F_{\varepsilon}$ is continuous. Therefore, by Schauder's fixed point theorem, $F_{\varepsilon}$ has at least one fixed point $\left(h_{\varepsilon}, \bar{u}_{\varepsilon}\right)$.

Step 7: The limit for $\varepsilon \rightarrow 0$

In order to pass to the limit as $\varepsilon \rightarrow 0$, we state the following:

Claim 1. For all $\tau>0$ small enough, there holds the estimates

$$
\begin{aligned}
& \int_{0}^{T^{*}} \int_{B_{2 M}} \chi_{\Omega_{h_{\varepsilon}^{\star}(t)}}\left|\bar{u}_{\mathcal{\varepsilon}}(t, \cdot)-\bar{u}_{\varepsilon}(t-\tau, \cdot)\right|^{2} d t \\
& +\int_{0}^{T^{*}} \int_{\omega}\left(\partial_{t} h(t, \cdot)-\partial_{t} h_{\varepsilon}(t-\tau, \cdot)\right)^{2} d t \leq C \sqrt{\tau}
\end{aligned}
$$

and

$$
\int_{0}^{T^{*}} \int_{B_{2 M}}\left|\chi_{\Omega_{h_{\varepsilon}^{\star}(t)}} \bar{u}_{\varepsilon}(t, \cdot)-\chi_{\Omega_{h_{\varepsilon}^{\star}(t-\tau)}} \bar{u}_{\varepsilon}(t-\tau, \cdot)\right|^{2} d t \leq C \sqrt{\tau}
$$

with $C>0$ independent of $\varepsilon$ and where $h_{\varepsilon}(t):=h_{0}^{\varepsilon}, \partial_{t} h_{\varepsilon}(t):=0$ and $\bar{u}_{\varepsilon}(t):=0$ if $t<0$.

Actually, since the proof of this claim is almost word for word the same as the one in [1], Lemma 9, we only treat here the new, additional term

$$
\int_{0}^{T^{*}} \int_{\omega} G_{\varepsilon}\left(\partial_{t} h_{\varepsilon}^{n}\right) b
$$

The key idea in the proof is to test the equation solved by $\left(u_{\varepsilon}, h_{\varepsilon}\right)$ with the special test function $\left(\phi_{\varepsilon}, b\right)$ depending on the solution $\left(h_{\varepsilon}, \bar{u}_{\varepsilon}\right)$ as follows:

$$
\phi_{\varepsilon}=\int_{t-\tau}^{t} u_{\varepsilon}^{[1+\delta]}(s, x, y,(1+\delta) z) d s, \quad b=\int_{t-\tau}^{t} \partial_{t} h_{\varepsilon}(s, x, y) d s
$$

for some $\delta>0$; see (23) for the definition of $u^{[1+\delta]}$. After inserting this test function, the resulting terms are estimated by bounds of the form $C \sqrt{\tau}$ with $C$ independent of $\varepsilon$. Here, the only new term is $\int_{0}^{T^{*}} \int_{\omega}\left(G_{\varepsilon}\left(\partial_{t} h_{\varepsilon}^{n}\right) \cdot \int_{t-\tau}^{t} \partial_{t} h_{\varepsilon}(s, x, y) d s\right)$, which we can estimate as follows

$$
\begin{aligned}
\mid \int_{0}^{T^{*}} & \int_{\omega}\left(G_{\varepsilon}\left(\partial_{t} h_{\varepsilon}^{n}\right) \cdot \int_{t-\tau}^{t} \partial_{t} h_{\varepsilon}(s, x, y) d s\right) \mid \\
& \leq \int_{0}^{T^{*}} \int_{\omega}\left|G_{\varepsilon}\left(\partial_{t} h_{\varepsilon}^{n}\right)\right|\left(\int_{0}^{T^{*}}\left|\partial_{t} h_{\varepsilon}(s, x, y)\right|^{2} d s\right)^{1 / 2} \sqrt{\tau} \\
& \leq C T^{*}\left\|\partial_{t} h_{\mathcal{\varepsilon}}\right\|_{L^{2}\left(0, T^{*} ; L^{2}(\omega)\right)} \sqrt{\tau} \leq C\left(T^{*}\right) \sqrt{\tau},
\end{aligned}
$$

where the uniform boundedness of $\left\|\partial_{t} h_{\varepsilon}\right\|_{L^{2}\left(0, T^{*} ; L^{2}(\omega)\right)}$ is used; see (50).

Next, we use the Fréchet-Kolmogorov theorem. Here, (56) and (57) imply the equicontinuity of $\left(\partial_{t} h_{\varepsilon}\right)_{\mathcal{\varepsilon}>0}$ and $\left(\chi_{\Omega_{h_{\varepsilon}^{\star}(t)}} \bar{u}_{\varepsilon}\right)_{\varepsilon>0}$ in $L^{2}\left(0, T^{*} ; L^{2}(\omega)\right)$ and $L^{2}\left(\left(0, T^{*}\right) \times B_{2 M}\right)$, respectively. Thus, we deduce that $\left(\partial_{t} h_{\varepsilon}\right)_{\varepsilon>0}$ is relatively compact in $L^{2}\left(\left(0, T^{*}\right) \times \omega\right)$ and $\left(\chi_{\Omega_{h_{\varepsilon}^{\star}(t)}} \bar{u}_{\varepsilon}\right)_{\varepsilon>0}$ is relatively compact in $L^{2}\left(0, T^{*} ; L^{2}\left(B_{2 M}\right)\right)$. This implies that $\left(\bar{u}_{\varepsilon}\right)_{\varepsilon>0}$ is relatively compact in $L^{2}\left(0, T^{*} ; L^{2}\left(B_{2 M}\right)\right)$.

From these considerations, as well as from the estimates in (50) where the constant does not depend on $\varepsilon$, we find functions $h$ on $\left[0, T^{*}\right] \times \bar{\omega}$ and $u$ on $\left[0, T^{*}\right] \times B_{2 M}$ such that the following convergences (of a suitable subsequence which we identify with the sequence itself) follow as $\varepsilon$ goes to 0 : 


$$
\begin{array}{cl}
h_{\varepsilon} \rightarrow h & \text { in } C^{0}\left(\left[0, T^{*}\right] \times \bar{\omega}\right), \\
h_{\varepsilon} \rightarrow h & \text { in } H^{1}\left(0, T^{*} ; H_{0}^{2}(\omega)\right), \\
\partial_{t} h_{\varepsilon} \rightarrow \partial_{t} h & \text { in } L^{2}\left(0, T^{*} ; L^{2}(\omega)\right), \\
\bar{u}_{\varepsilon} \rightarrow u & \text { in } L^{2}\left(0, T^{*} ; L^{2}\left(B_{2 M}\right)\right), \\
\chi_{\Omega_{h_{\varepsilon}^{\star}(t)} u_{\varepsilon}} \rightarrow \chi_{\Omega_{h(t)}} u & \text { in } L^{2}\left(0, T^{*} ; L^{2}\left(B_{2 M}\right)\right), \\
\bar{u}_{\varepsilon} \rightarrow u & \text { in } L^{2}\left(0, T^{*} ; H_{0, \Gamma_{0}}^{1}\left(B_{2 M}\right)\right), \\
h_{\varepsilon}^{\star} \rightarrow h & \text { in } C^{0}\left(\left[0, T^{*}\right] \times \bar{\omega}\right), \\
\partial_{t} h_{\varepsilon}^{\star} \rightarrow \partial_{t} h & \text { in } L^{2}\left(0, T^{*} ; L^{2}(\omega)\right), \\
\bar{u}_{\varepsilon}^{\star} \rightarrow u & \text { in } L^{2}\left(0, T^{*} ; L^{2}\left(B_{2 M}\right)\right) .
\end{array}
$$

The last three convergences follow from the way we regularized $h_{\varepsilon}$ and $u_{\varepsilon}$, as in (24) and (27), and from the corresponding convergences without regularization. Furthermore, $\left(\chi_{\Omega_{h_{\varepsilon}^{\star}(t)}} \nabla \bar{u}_{\varepsilon}\right)_{\mathcal{E}}>0$ converges (up to a subsequence) weakly to a matrix-valued function $q \in$ $\left(L^{2}\left(0, T ; L^{2}\left(B_{2 M}\right)\right)\right)^{3 \times 3}$ due to $(50)$ as $\varepsilon \rightarrow 0$.

Let $\varphi \in\left(L^{2}\left(\left(0, T^{*}\right) \times B_{2 M}\right)\right)^{3 \times 3}$ such that supp $\varphi \subset \bigcup_{t \in\left(0, T^{*}\right)}\{t\} \times\left(B_{2 M} \backslash \Omega_{h(t)}\right)$. Then, we have

$$
\begin{aligned}
& \left|\int_{\left(0, T^{*}\right) \times B_{2 M}} \chi_{\Omega_{h_{\varepsilon}^{\star}(t)}} \nabla \bar{u}_{\varepsilon}: \varphi\right|=\left|\int_{\left(0, T^{*}\right) \times B_{2 M}} \chi_{\Omega_{h_{\varepsilon}^{\star}(t)}} \nabla \bar{u}_{\varepsilon}: \varphi\left(\chi_{\Omega_{h_{\varepsilon}^{\star}(t)}}-\chi_{\Omega_{h(t)}}\right)\right| \\
& \quad \leq\left\|\chi_{\Omega_{h_{\varepsilon}^{\star}(t)}} \nabla \bar{u}_{\varepsilon}\right\|_{L^{2}\left(\left(0, T^{*}\right) \times B_{2 M}\right)}\left\|\varphi\left(\chi_{\Omega_{h_{\varepsilon}^{\star}(t)}}-\chi_{\Omega_{h(t)}}\right)\right\|_{L^{2}\left(\left(0, T^{*}\right) \times B_{2 M}\right)} \\
& \quad \stackrel{(50)}{\leq} C \| \varphi\left(\chi_{\Omega_{h_{\varepsilon}^{\star}(t)}}-\chi_{\left.\Omega_{h(t)}\right)} \|_{L^{2}\left(\left(0, T^{*}\right) \times B_{2 M}\right)} \longrightarrow 0 \text { as } \varepsilon \rightarrow 0\right.
\end{aligned}
$$

since $h_{\varepsilon}^{\star}$ converges to $h$ in $C^{0}([0, T] \times \bar{\omega})$ as $\varepsilon \rightarrow 0$. This implies that $q=0$ on $\bigcup_{t \in\left(0, T^{*}\right)}\{t\} \times$ $\left(B_{2 M} \backslash \Omega_{h(t)}\right)$.

Furthermore, let $\psi \in L^{2}\left(0, T^{*} ; H_{0, \Gamma_{0}}^{1}\left(B_{2 M}\right)\right)$ with supp $\psi \subset \bigcup_{t \in\left(0, T^{*}\right)}\{t\} \times \Omega_{h(t)}$. In a similar fashion as before, we have

$$
\begin{aligned}
& \left|\int_{\left(0, T^{*}\right) \times B_{2 M}}\left(\nabla \bar{u}_{\varepsilon}-\chi_{\Omega_{h_{\varepsilon}^{\star}(t)}} \nabla \bar{u}_{\varepsilon}\right): \nabla \psi\right| \\
& \quad \leq\left\|\nabla \overline{\mathcal{u}}_{\varepsilon}\right\|_{L^{2}\left(\left(0, T^{*}\right) \times B_{2 M}\right)}|| \mid \chi_{\Omega_{h(t)}-\chi_{\Omega_{h_{\varepsilon}^{\star}(t)}}|| \nabla \psi \mid \|_{L^{2}\left(\left(0, T^{*}\right) \times B_{2 M}\right)}} \\
& \quad \leq C \| \mid \chi_{\Omega_{h(t)}-\chi_{\Omega_{h_{\varepsilon}^{\star}(t)}}|| \nabla \psi \mid \|_{L^{2}\left(\left(0, T^{*}\right) \times B_{2 M}\right)} \longrightarrow 0 \text { as } \varepsilon \rightarrow 0 .}
\end{aligned}
$$

Therefore, $q=\nabla u$ on $\bigcup_{t \in\left(0, T^{*}\right)}\{t\} \times \Omega_{h(t)}$ and, in total,

$$
\chi_{\Omega_{h_{\varepsilon}^{\star}(t)}} \nabla \bar{u}_{\varepsilon} \rightarrow q=\chi_{\Omega_{h(t)}} \nabla u \text { in } L^{2}\left(0, T^{*} ; L^{2}\left(B_{2 M}\right)\right) .
$$

Next, we proceed to the limit in the equation

$$
u_{\mathcal{\varepsilon}}\left(t, x, y, 1+h_{\mathcal{\varepsilon}}^{\star}(t, x, y)\right)=\left(0,0, \partial_{t} h_{\mathcal{\varepsilon}}(t, x, y)\right)^{\top} \text { on }\left[0, T^{*}\right] \times \omega .
$$

We already know that the right-hand side converges to $\left(0,0, \partial_{t} h\right)^{\top}$ in $L^{2}\left(0, T^{*} ; L^{2}(\omega)\right)$. We consider

$$
w_{\varepsilon}= \begin{cases}\left(0,0, \partial_{t} h_{\varepsilon}\right)^{\top} & \text { in } B_{2 M} \backslash Z_{m / 2} \\ z_{\partial_{t}} h_{\varepsilon} & \text { in } Z_{m / 2}\end{cases}
$$


with $z_{\partial_{t}} h_{\varepsilon} \in L^{2}\left(0, T^{*} ; H_{0, \Gamma_{0}}^{1}\left(Z_{m / 2}\right)\right)$ solving (55) but with $b$ replaced with $\partial_{t} h_{\varepsilon}$. Then, $\left(\bar{u}_{\varepsilon}-\right.$ $\left.w_{\varepsilon}\right)_{\varepsilon>0}$ is bounded in $L^{2}\left(0, T^{*} ; H^{1}\left(B_{2 M}\right)\right)$ with a constant independent of $\varepsilon$. This and (58) imply that a subsequence of $\left(\bar{u}_{\varepsilon}-w_{\varepsilon}\right)_{\varepsilon}>0$ converges weakly to $w_{0}:=u-w$ with

$$
w:= \begin{cases}\left(0,0, \partial_{t} h\right)^{\top} & \text { in } B_{2 M} \backslash Z_{m / 2} \\ z_{\partial_{t} h} & \text { in } Z_{m / 2}\end{cases}
$$

in $L^{2}\left(0, T^{*} ; H^{1}\left(B_{2 M}\right)\right)$. Let $\delta>0$ be arbitrary. Due to the uniform convergence of $\left(h_{\varepsilon}^{\star}\right)_{\varepsilon>0}$ to $h$, we get that

$$
\exists \tilde{\varepsilon}>0 \forall \varepsilon \in(0, \tilde{\varepsilon}] \forall t \in\left(0, T^{*}\right): \Omega_{h(t)+\delta} \supset \Omega_{h_{\varepsilon}(t)} .
$$

This implies that

$$
\bar{u}_{\varepsilon}=\left(0,0, \partial_{t} h_{\varepsilon}\right)^{\top} \text { on } B_{2 M} \backslash \Omega_{h(t)+\delta} \subset B_{2 M} \backslash \Omega_{h_{\varepsilon}(t)}
$$

for all $\varepsilon \in(0, \tilde{\varepsilon}]$ and $t \in\left(0, T^{*}\right)$. We also have that

$$
w_{\varepsilon}=\left(0,0, \partial_{t} h_{\varepsilon}\right)^{\top} \text { on } B_{2 M} \backslash \Omega_{h(t)+\delta} \subset B_{2 M} \backslash Z_{m / 2}
$$

because of $1+h \geq m$ on $\left[0, T^{*}\right] \times \omega$. Since $\delta>0$ was arbitrary, we deduce that $w_{0}=0$ in $\bigcup_{t \in\left(0, T^{*}\right)}\{t\} \times\left(B_{2 M} \backslash \Omega_{h(t)}\right)$. Due to $1+h_{\varepsilon}^{\star} \geq \frac{m}{2}$ on $\left[0, T^{*}\right] \times \omega$, we also deduce that $w_{0}=0$ on $\Gamma_{0}$. This readily implies that

$$
w_{0}=0 \text { in } \bigcup_{t \in\left(0, T^{*}\right)}\{t\} \times\left(B_{2 M} \backslash \Omega_{h(t)}\right) \text { and } w_{0} \in L^{2}\left(0, T^{*} ; H_{0}^{1}\left(\Omega_{h(t)}\right)\right) .
$$

Due to Lemma 2, it follows that $\gamma_{h(t)}\left(w_{0}\right)=0$. Since $\gamma_{h(t)}(w)=\left(0,0, \partial_{t} h\right)^{\top}$, it must be the case that

$$
\gamma_{h(t)}(u)=\left(0,0, \partial_{t} h\right)^{\top} \text {. }
$$

This shows that the interface condition is satisfied.

Now, we take the limit in the equation solved by $\left(u_{\varepsilon}, h_{\varepsilon}\right)$. Recall that $\left(u_{\varepsilon}, h_{\varepsilon}\right)$ solves

$$
\begin{gathered}
\int_{0}^{t} \int_{\Omega_{h_{\varepsilon}^{\star}(s)}} \partial_{t} u_{\varepsilon} \cdot \phi_{\varepsilon}+\mu \int_{0}^{t} \int_{\Omega_{h_{\varepsilon}^{\star}(s)}} \nabla u_{\varepsilon}: \nabla \phi_{\varepsilon}+\frac{1}{2} \int_{0}^{t} \int_{\Omega_{h_{\varepsilon}^{\star}(s)}}\left(u_{\varepsilon}^{\star} \cdot \nabla\right) u_{\varepsilon} \cdot \phi_{\varepsilon} \\
\quad-\frac{1}{2} \int_{0}^{t} \int_{\Omega_{h_{\varepsilon}^{\star}(s)}}\left(u_{\varepsilon}^{\star} \cdot \nabla\right) \phi_{\varepsilon} \cdot u_{\varepsilon}+\frac{1}{2} \int_{0}^{t} \int_{\omega} \partial_{t} h_{\varepsilon}\left(\partial_{t} h_{\varepsilon}^{\star}\right) b+\int_{0}^{t} \int_{\omega} \partial_{t}^{2} h_{\varepsilon} b \\
\quad+\int_{0}^{t} \int_{\omega} \Delta \partial_{t} h_{\varepsilon} \Delta b+\int_{0}^{t} \int_{\omega} \Delta h_{\varepsilon} \Delta b+\int_{0}^{t} \int_{\omega} \kappa G_{\varepsilon}\left(\partial_{t} h_{\varepsilon}\right) b=\int_{0}^{t} \int_{\Omega_{h_{\varepsilon}^{\star}(s)}} f \cdot \phi_{\varepsilon}
\end{gathered}
$$

for all $\phi_{\varepsilon} \in V_{h_{\varepsilon}^{\star}}, b \in L^{2}\left(0, T ; H_{0}^{2}(\omega)\right)$ such that $\phi_{\varepsilon}\left(t, x, y, 1+h_{\varepsilon}^{\star}(t, x, y)\right)=(0,0, b(t, x, y))^{\top}$ on $\left[0, T^{*}\right] \times \omega$. This equation is derived from (28) by replacing $(v, \delta)$ with the fixed point $\left(u_{\varepsilon}, h_{\varepsilon}\right)$ found in Section 6. In particular, $\delta_{\varepsilon}^{\star}=N_{\varepsilon}(\delta)$ becomes $N_{\varepsilon}\left(h_{\varepsilon}\right)=h_{\varepsilon}^{\star}$ and $v_{\varepsilon}^{\star}=R_{\varepsilon}(v)$ becomes $R_{\varepsilon}\left(u_{\varepsilon}\right)=u_{\varepsilon}^{\star}$, but note that $u_{\varepsilon}$ and $h_{\varepsilon}$ will still differ from $u_{\varepsilon}^{\star}$ and $h_{\varepsilon}^{\star}$, respectively. Since $G_{\varepsilon}$ is the derivative of the convex function $j_{\varepsilon}: \mathbb{R} \rightarrow \mathbb{R}, x \mapsto \sqrt{x^{2}+\varepsilon^{2}}$, we get

$$
-G_{\varepsilon}\left(\partial_{t} h_{\varepsilon}\right) b=G_{\varepsilon}\left(\partial_{t} h_{\varepsilon}\right)\left(-b+\partial_{t} h_{\varepsilon}-\partial_{t} h_{\varepsilon}\right) \leq \sqrt{\left(\partial_{t} h_{\varepsilon}-b\right)^{2}+\varepsilon^{2}}-\sqrt{\partial_{t} h_{\varepsilon}^{2}+\varepsilon^{2}},
$$

which is equivalent to

$$
\sqrt{\partial_{t} h_{\varepsilon}^{2}+\varepsilon^{2}}-\sqrt{\left(\partial_{t} h_{\varepsilon}-b\right)^{2}+\varepsilon^{2}} \leq G_{\varepsilon}\left(\partial_{t} h_{\varepsilon}\right) b
$$


If we plug this into (60) and use integration by parts with respect to time for the first term and for the term involving $\partial_{t}^{2} h_{\varepsilon}$, as in (30) and (29) (with $\delta$ replaced by $h$ ), we end up with the inequality

$$
\begin{aligned}
& \int_{\Omega_{h_{\varepsilon}^{\star}(t)}} u_{\varepsilon}(t) \cdot \phi_{\varepsilon}(t)-\int_{0}^{t} \int_{\Omega_{h_{\varepsilon}^{\star}(s)}} u_{\varepsilon} \cdot \partial_{t} \phi_{\varepsilon}+\mu \int_{0}^{t} \int_{\Omega_{h_{\varepsilon}^{\star}(s)}} \nabla u_{\varepsilon}: \nabla \phi_{\varepsilon} \\
& \quad+\frac{1}{2} \int_{0}^{t} \int_{\Omega_{h_{\varepsilon}^{\star}(s)}}\left(u_{\varepsilon}^{\star} \cdot \nabla\right) u_{\varepsilon} \cdot \phi_{\varepsilon}-\frac{1}{2} \int_{0}^{t} \int_{\Omega_{h_{\varepsilon}^{\star}(s)}}\left(u_{\varepsilon}^{\star} \cdot \nabla\right) \phi_{\varepsilon} \cdot u_{\varepsilon}-\frac{1}{2} \int_{0}^{t} \int_{\omega} \partial_{t} h_{\varepsilon}\left(\partial_{t} h_{\varepsilon}^{\star}\right) b \\
& \quad+\int_{\omega} \partial_{t} h_{\varepsilon}(t) b(t)-\int_{0}^{t} \int_{\omega} \partial_{t} h_{\varepsilon} \partial_{t} b+\int_{0}^{t} \int_{\omega} \Delta \partial_{t} h_{\varepsilon} \Delta b+\int_{0}^{t} \int_{\omega} \Delta h_{\varepsilon} \Delta b \\
& \quad+\int_{0}^{t} \int_{\omega} \kappa\left(\sqrt{\partial_{t} h_{\varepsilon}^{2}+\varepsilon^{2}}-\sqrt{\left(\partial_{t} h_{\varepsilon}-b\right)^{2}+\varepsilon^{2}}\right) \\
& \leq \int_{0}^{t} \int_{\Omega_{h_{\varepsilon}^{\star}(s)}} f \cdot \phi_{\varepsilon}+\int_{\omega} h_{1}^{\varepsilon} b(0)+\int_{\Omega_{h_{0}^{\varepsilon}}} u_{0}^{\varepsilon} \phi_{\varepsilon}(0)
\end{aligned}
$$

for a.e. $t \in\left(0, T^{*}\right)$ and for all $\left(\phi_{\varepsilon}, b\right) \in \mathcal{V}_{h_{\varepsilon}^{\star}} \times C^{1}\left(\left[0, T^{*}\right] ; H_{0}^{2}(\omega)\right)$ with $\phi_{\varepsilon}\left(t, x, y, 1+h_{\varepsilon}^{\star}\right)=$ $(0,0, b)^{\top}$ on $[0, T] \times \omega$.

As in the variational problem (54), we have to deal with the problem that the test functions depend on $\varepsilon$. We solve this issue in a similar manner and consider

$$
\phi^{0} \in C_{c}^{\infty}\left(\bigcup_{t \in\left(0, T^{*}\right)}\{t\} \times \Omega_{h(t)}\right), \operatorname{div} \phi^{0}=0 .
$$

Then, $\left(\phi^{0}, 0\right)$ is an admissible test function for small $\varepsilon$ since $\phi^{0} \in \mathcal{V}_{h_{\varepsilon}^{\star}}$ for $\varepsilon>0$ sufficiently small due to the uniform convergence of $h_{\varepsilon}^{\star}$ to $h$ as $\varepsilon$ goes to 0 . For an element $b \in$ $C^{1}\left(\left[0, T^{*}\right] ; H_{0}^{2}(\omega)\right)$ with $\int_{\omega} b=0$, we use the continuous embedding $H^{2}(\omega) \hookrightarrow W_{4}^{\frac{3}{4}}(\omega)$ to deduce that there exists a $z \in L^{\infty}\left(0, T^{*} ; W_{4}^{1}\left(Z_{m / 2}\right)\right) \subset L^{\infty}\left(\left(0, T^{*}\right) \times Z_{m / 2}\right)$ such that

$$
\operatorname{div} z=0 \text { in }\left(0, T^{*}\right) \times Z_{m / 2}, \quad z_{\mid \partial Z_{m / 2}}= \begin{cases}(0,0, b)^{\top} & \text { on }\left(0, T^{*}\right) \times \omega \times\left\{\frac{m}{2}\right\}, \\ 0 & \text { else } .\end{cases}
$$

Then, we consider

$$
\phi^{1}(b):= \begin{cases}(0,0, b)^{\top} & \text { on } B_{2 M} \backslash Z_{m / 2} \\ z & \text { on } Z_{m / 2} .\end{cases}
$$

The pair $\left(\phi^{1}(b), b\right)$ is an admissible test function, too, since $1+\delta_{\varepsilon}^{\star} \geq \frac{m}{2}$ on $\left(0, T^{*}\right) \times \omega$ for all $\varepsilon>0$. For this class of test functions, we can go to the limit in (61) due to the convergences (58) and (59). Here, let us consider the most problematic term

$$
\begin{aligned}
& \int_{0}^{t} \int_{\Omega_{h_{\varepsilon}^{\star}(s)}}\left(u_{\varepsilon}^{\star} \cdot \nabla\right) u_{\varepsilon} \cdot \phi=\int_{0}^{t} \int_{B_{2 M}} \chi_{\Omega_{h_{\varepsilon}^{\star}(s)}}\left(u_{\varepsilon}^{\star} \cdot \nabla\right) u_{\varepsilon} \cdot \phi \\
& =\int_{0}^{t} \int_{B_{2 M}} \chi_{\Omega_{h_{\varepsilon}^{\star}(s)}}(u \cdot \nabla) u_{\varepsilon} \cdot \phi+\int_{0}^{t} \int_{B_{2 M}} \chi_{\Omega_{h_{\varepsilon}^{\star}(s)}}\left(\left(u_{\varepsilon}^{\star}-u\right) \cdot \nabla\right) u_{\varepsilon} \cdot \phi .
\end{aligned}
$$

The first term on the right-hand side converges to $\int_{0}^{t} \int_{\Omega_{h(s)}}(u \cdot \nabla) u \cdot \phi$, the correct limit due to (59), and the second summand converges to 0 because of the strong convergence $\bar{u}_{\varepsilon}^{\star} \rightarrow u$ in $L^{2}\left(0, T^{*} ; L^{2}\left(B_{2 M}\right)\right)$ and the boundedness of $\left(\chi_{\Omega_{h_{\varepsilon}^{*}(s)}} \nabla u_{\varepsilon}\right)_{\varepsilon>0}$ in $L^{2}\left(0, T^{*} ; L^{2}\left(B_{2 M}\right)\right)$. Note that this argument works since $\left.\phi \in L^{\infty}\left(\left(0, T^{*}\right) \times Z_{m / 2}\right)\right)$; this, in retrospect, explains why 
the previous divergence problem was solved for an exponent larger than 2. Concerning the term $\int_{0}^{t} \int_{\Omega_{h_{\varepsilon}^{\star}(s)}}\left(u_{\varepsilon}^{\star} \cdot \nabla\right) \phi \cdot u_{\varepsilon}$, we estimate

$$
\begin{aligned}
& \int_{0}^{t} \int_{B_{2 M}}\left|\chi_{\Omega_{h_{\varepsilon}^{\star}(s)}}\left(u_{\varepsilon}^{\star} \cdot \nabla\right) \phi \cdot u_{\varepsilon}-\chi_{\Omega_{h(s)}}(u \cdot \nabla) \phi \cdot u\right| \\
& \left.\leq \int_{0}^{t} \int_{B_{2 M}} \mid\left(\chi_{\Omega_{h_{\varepsilon}^{\star}(s)}} u_{\varepsilon}^{\star}-\chi_{\Omega_{h(s)}} u\right) \cdot \nabla\right) \phi \cdot \chi_{\Omega_{h_{\varepsilon}^{\star}(s)}} u_{\varepsilon} \mid \\
& \quad+\int_{0}^{t} \int_{B_{2 M}}\left|\left(\chi_{\Omega_{h(s)}} u \cdot \nabla\right) \phi \cdot\left(\chi_{\Omega_{h(s)}} u-\chi_{\Omega_{h_{\varepsilon}^{\star}(s)}} u_{\varepsilon}\right)\right| \\
& \leq\left\|\chi_{\Omega_{h_{\varepsilon}^{\star}(s)}} u_{\varepsilon}^{\star}-\chi_{\Omega_{h(s)}} u\right\|_{L^{2}\left((0, t) \times B_{2 M}\right)}\|\nabla \phi\|_{L^{\infty}\left(0, t ; L^{4}\left(B_{2 M}\right)\right)} \\
& \quad \cdot\left(\left\|\bar{u}_{\varepsilon}\right\|_{L^{2}\left(0, t ; L^{4}\left(B_{2 M}\right)\right)}+\left\|\chi_{\Omega_{h(s)}} u\right\|_{L^{2}\left(0, t ; L^{4}\left(B_{2 M}\right)\right)}\right) \\
& \left.\quad+\left\|\chi_{\Omega_{h_{\varepsilon}^{\star}(s)}}\left(u_{\varepsilon}^{\star}-u_{\varepsilon}\right)\right\|_{L^{2}\left((0, t) \times B_{2 M}\right)}\|\nabla \phi\|_{L^{\infty}\left(0, t ; L^{4}\left(B_{2 M}\right)\right)}\left\|\chi_{\Omega_{h(s)}} u\right\|_{L^{2}\left(0, t ; L^{4}\left(B_{2 M}\right)\right)}\right),
\end{aligned}
$$

which converges to 0 as $\varepsilon \rightarrow 0$ due to the boundedness of $\left(\bar{u}_{\varepsilon}\right)_{\varepsilon}$ in $L^{2}\left(0, t ; L^{4}\left(B_{2 M}\right)\right)$ implied by $(58)_{6}$; the term $\chi_{\Omega_{h_{\varepsilon}^{\star}(s)}}\left(u_{\varepsilon}^{\star}-u_{\varepsilon}\right)$ converges to 0 in $L^{2}$ by $(58)_{4,9}$ and the triangle inequality. For the convergence of the non-linear boundary term in (61), due to

$$
\partial_{t} h_{\varepsilon} \rightarrow \partial_{t} h \text { in } L^{2}\left(0, T ; L^{2}(\omega)\right) \hookrightarrow L^{1}\left(0, T ; L^{1}(\omega)\right) \text { as } \varepsilon \rightarrow 0
$$

and $\sqrt{x^{2}+\varepsilon^{2}} \leq|x|+\varepsilon$ for $x \in \mathbb{R}$, the dominated convergence theorem implies that

$$
\int_{0}^{t} \int_{\omega}\left(\sqrt{\partial_{t} h_{\varepsilon}^{2}+\varepsilon^{2}}-\sqrt{\left(\partial_{t} h_{\varepsilon}-b\right)^{2}+\varepsilon^{2}}\right) \rightarrow \int_{0}^{t} \int_{\omega}\left(\left|\partial_{t} h\right|-\left|\partial_{t} h-b\right|\right) \text { as } \varepsilon \rightarrow 0 .
$$

This shows that $(u, h)$ is a solution of (20) for these special test functions.

Now, for a general test function $(\phi, b) \in \mathcal{V}_{h} \times C^{1}\left(\left[0, T^{*}\right] ; H_{0}^{2}(\omega)\right)$ with $\phi(t, x, y, 1+$ $h(t, x, y))=(0,0, b(t, x, y))$ in $[0, T] \times \omega$, we write $\phi=\left(\phi-\phi^{1}(b)\right)+\phi^{1}(b)$ with $\phi^{1}(b)$ as before. Since the Dirichlet part $\phi-\phi^{1}(b)$ can be approximated by functions of the same type as $\phi^{0}$, we can recover the weak formulation (20) for general test functions. This shows that $(u, h)$ is a weak solution of (2)-(9), which concludes the proof.

\section{Discussion}

We presented the existence of weak solutions to a fluid-structure interaction problem for Navier-Stokes equations in a domain with a damped Kirchhoff plate as an upper lid. The interaction is given only in the normal direction, together with a threshold for the normal stress, so that, for small stresses, the plate stays immovable. The proof follows the line of Reference [1], where the damped plate is linearly coupled with the fluid. There appear the natural questions whether similar results can be obtained for unbounded domains of half space type and for bounded domains where the plate is fastened to the arbitrarily shaped upper brim of the lateral boundary of a cylindrical domain or even cannot be written as a graph.

Author Contributions: This article is part of the PhD thesis of A.S. [18]. Writing, review and editing were carried out by both authors in equal parts. All authors have read and agreed to the published version of the manuscript.

Funding: This research received no external funding.

Institutional Review Board Statement: Not applicable.

Informed Consent Statement: Not applicable.

Conflicts of Interest: The authors declare no conflict of interest. 


\section{References}

1. Chambolle, A.; Desjardins, B.; Esteban, M.J.; Grandmont, C. Existence of weak solutions for the unsteady interaction of a viscous fluid with an elastic plate. J. Math. Fluid Mech. 2005, 7, 368-404. [CrossRef]

2. Grandmont, C. Existence of weak solutions for the unsteady interaction of a viscous fluid with an elastic plate. SIAM J. Math. Anal. 2008, 40, 716-737. [CrossRef]

3. Denk, R.; Saal, J. $L^{p}$-theory for a fluid-structure interaction model. Z. Angew. Math. Phys. 2020, 71, 158. [CrossRef]

4. Celik, A.; Kyed, M. Fluid-plate interaction under periodic forcing. arXiv 2021, arXiv:2103.00795.

5. da Veiga, H.B. On the existence of strong solutions to a coupled fluid-structure evolution problem. J. Math. Fluid Mech. 2004, 6, 21-52. [CrossRef]

6. Badra, M.; Takahashi, T. Gevrey regularity for a system coupling the Navier-Stokes system with a beam equation. SIAM J. Math. Anal. 2019, 51, 4776-4814. [CrossRef]

7. Chueshov, I.; Ryzhkova, I. Unsteady interaction of a viscous fluid with an elastic shell modeled by full von Karman equations. J. Differ. Equ. 2013, 254, 1833-1862. [CrossRef]

8. Lengeler, D.; Růžička, R. Weak solutions for an incompressible Newtonian fluid interacting with a Koiter type shell. Arch. Ration. Mech. Anal. 2014, 211, 205-255. [CrossRef]

9. Kashiwabara, T. On a strong solution of the non-stationary Navier-Stokes equations under slip or leak boundary conditions of friction type. J. Differ. Equ. 2013, 254, 756-778. [CrossRef]

10. Saito, N. On the Stokes Equation with the Leak and Slip Boundary Conditions of Friction Type: Regularity of Solutions. Publ. Res. Inst. Math. Sci. 2004, 40, 345-383. [CrossRef]

11. Bălilescu, L.; San Martín, J.; Takahashi, T. On the Navier-Stokes system with the Coulomb friction law boundary condition. Z. Angew. Math. Phys. 2017, 68, 1-25. [CrossRef]

12. Jing, F.; Li, J.; Chen, Z.; Yan, W. Discontinuous Galerkin methods for the incompressible flow with nonlinear leak boundary conditions of friction type. Appl. Math. Lett. 2017, 73, 113-119. [CrossRef]

13. Bălilescu, L.; San Martín, J.; Takahashi, T. Fluid-rigid structure interaction system with Coulomb's law. SIAM J. Math. Anal. 2017, 49, 4625-4657. [CrossRef]

14. Consiglieri, L. A nonlocal friction problem for a class of non-Newtonian flows. Port. Math. 2003, 60, $237-252$.

15. Baranovskii, E.S. Steady flows of an Oldroyd fluid with threshold slip. Commun. Pure Appl. Anal. 2019, 18, 735-750. [CrossRef]

16. Haupt, P. Continuum Mechanics and Theory of Materials; Advanced Texts in Physics; Springer: Berlin, Germany, 2000.

17. Adams, R.A.; Fournier, J.J.F. Sobolev Spaces, 2nd ed.; Pure and Applied Mathematics (Amsterdam) 140; Elsevier/Academic Press: Amsterdam, The Netherlands, 2003.

18. Schmidt, A. The Navier-Stokes Equations with Elastic Boundary and Boundary Conditions of Friction Type. Ph.D. Thesis, Darmstadt University of Technology, Darmstadt, Germany, 2021.

19. Temam, R. Navier-Stokes Equations: Theory and Numerical Analysis; North-Holland Publishing Company: Amsterdam, The Netherlands, 1977.

20. Gilbarg, D.; Trudinger, N.S. Elliptic Partial Differential Equations of Second Order; Grundlehren der Mathematischen Wissenschaften Volume 224; Springer: Berlin, Germany; New York, NY, USA, 1977. 